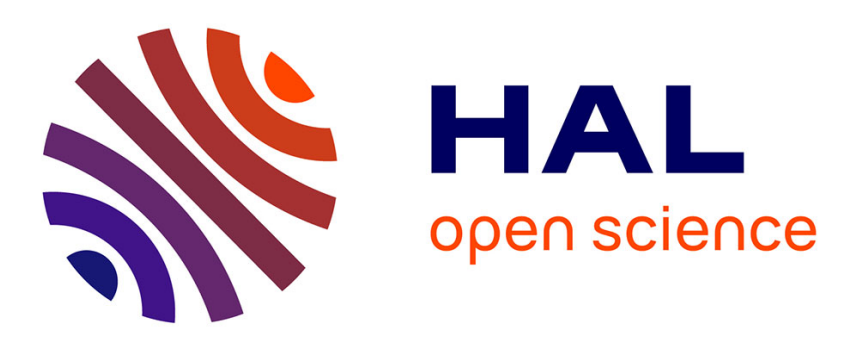

\title{
Medium-frequency electromagnetic device to measure electric conductivity and dielectric permittivity of soils
}

Pauline Kessouri, Sébastien Flageul, Quentin Vitale, Solène Buvat, Fayçal Rejiba, Alain Tabbagh

\section{- To cite this version:}

Pauline Kessouri, Sébastien Flageul, Quentin Vitale, Solène Buvat, Fayçal Rejiba, et al.. Mediumfrequency electromagnetic device to measure electric conductivity and dielectric permittivity of soils. Geophysics, 2016, 81 (1), pp.E1-E16. 10.1190/geo2014-0468.1 . hal-01376575

\section{HAL Id: hal-01376575 https://hal.sorbonne-universite.fr/hal-01376575}

Submitted on 5 Oct 2016

HAL is a multi-disciplinary open access archive for the deposit and dissemination of scientific research documents, whether they are published or not. The documents may come from teaching and research institutions in France or abroad, or from public or private research centers.
L'archive ouverte pluridisciplinaire HAL, est destinée au dépôt et à la diffusion de documents scientifiques de niveau recherche, publiés ou non, émanant des établissements d'enseignement et de recherche français ou étrangers, des laboratoires publics ou privés. 
Medium frequency electromagnetic device to measure electrical conductivity and dielectric permittivity of soils

\author{
P. Kessouri(1,2), S. Flageul(2), Q. Vitale(2), S. Buvat(3), F. Rejiba(2), A. Tabbagh(2)
}

(1) Colorado School of Mines, Department of Geophysics, Golden, 80401, CO, USA

(2) Sorbonne-Universités, UPMC/CNRS, UMR 7619, Métis, Paris, France

(3) Institut des Sciences de la Terre d'Orléans, 1A, rue de la Férollerie, F-45071 Orléans Cedex 2,

France

(1)

Corresponding Author: Pauline Kessouri (kessouri@mines.edu)

Emails: sebastien.flageul@upmc.fr; quentin.vitale@upmc.fr; $\underline{\text { solene.buvat@upmc.fr; }}$

faycal.rejiba@upmc.fr; alain.tabbagh@upmc.fr

5

6 Running Title: Medium frequency EM prototype 


\section{Abstract}

An electromagnetic tool working in the medium frequency range allows the determination of both electrical conductivity and dielectric permittivity of soils with a single measurement. It brings information about different state parameters of soils, especially their water and clay contents for a significant volume of investigation. To investigate these properties, a medium frequency range EM prototype, the CE120, was built using a PERP (perpendicular coils) Slingram configuration with a working frequency of $1.56 \mathrm{MHz}$ and a fixed coil spacing of $1.2 \mathrm{~m}$. This configuration was chosen using modeling with the purpose of measuring electrical resistivities up to a few thousands ohm-m and relative dielectric permittivities as low as 2 . These thresholds match the expected parameters in the medium frequency range. Moreover, the CE120 characteristics allow for an investigation depth between 2 and $2.5 \mathrm{~m}$, depending on the nature of the soil. The prototype was tested on two different soils: sandy alluvia and clay-loam soil. The electrical conductivities of the sandy alluvia can reach $10000 \Omega \mathrm{m}$, which is close to the detection threshold of the CE120. Consequently, the measured dielectric permittivity only includes high frequency effects (dielectric polarization) and can be converted to apparent volumetric water content. For the clay-loam soil, both the electrical conductivity and dielectric permittivity are measured and the volumetric water content in this case is obtained using an empirical relationship previously established in the laboratory on known samples. In both cases, the obtained results are coherent with the direct mass water content measurements.

Key-Words: EM prototype, medium frequency range, dielectric permittivity, electrical conductivity, water content, clay content 


\section{Introduction}

The electrical and magnetic properties, such as the electrical conductivity, the dielectric permittivity and the magnetic susceptibility are frequently used to estimate different soil state parameters (Friedman, 2005; Liu et al., 2012). These electrical properties can be measured by electromagnetic prospection devices. Depending on their working frequencies, different kinds of instruments have been developed. For the past fifty years, only EMI (Electro-Magnetic Induction) devices working in the low frequency (LF) range and ground penetrating RADAR (GPR) using high frequencies (HF) have been employed. In the low frequency range, the EMI instruments measure: (i) the soil electrical conductivity $\sigma$, which is strongly related to the soil water content, texture and clay content; (ii) the soil magnetic susceptibility $\kappa$, which is mainly linked to the different pedogenetic processes. Most of the devices use a Slingram geometry with separated transmitter and receiver coils and respect the low induction number (LIN) approximation. Both their investigation depth and their lateral resolution are determined by their geometrical parameters (mostly coil orientation and intercoil spacing). In this frequency range, polarization processes occurring in the ground cannot be measured due to the dominant conduction processes, with the exception of measurements taken over very resistive terrains (Huang and Fraser, 2002). In the high frequency range, dipolar polarization processes, due to water presence, dominate. The measured dielectric permittivity $\varepsilon$ is thus directly linked with the soil volumetric water content. This frequency range offers high resolution, but measurements cannot be performed on conductive soils, such as clay rich soils, where attenuation is significant (Walther et al., 1986; Knight, 2001).

The interest in developing devices working in the medium frequency (MF) range is quite new but presents different kinds of advantages, especially regarding the estimation of both soil water and clay content. To the best of our knowledge, no commercial EM device working in the medium frequency range has been produced and only two other prototypes 
were built (Stewart et al., 1994; Bourgeois et Lenain, 2002). This situation is explained by the difficulties encountered in the measurements interpretation: (i) no simplification of the Maxwell equations can be made; (ii) the electrical parameters both depend on the real and imaginary parts of the measured magnetic fields; (iii) the electrical conductivity and the dielectric permittivity are dispersive. An inversion procedure is thus needed to deduce the electrical conductivity and the dielectric permittivity from the complex magnetic fields. Tabbagh (1994), Stewart et al. (1994) and Bourgeois and Lenain (2002) all developed their own forward modeling and inversion schemes include the dielectric permittivity in the calculations. Yet, none of the proposed modeling takes into account the dispersive characters of the electrical properties: the electrical conductivity and the dielectric permittivity are assumed to be constant at all frequencies.

Both Stewart et al. (1994) and Bourgeois and Lenain (2002) choose to adapt the wellknown Slingram geometry in EMI for their prototypes. They could work at variable frequencies and spacings, allowing their use for 1D soundings and/or profiles. Stewart et al. (1994) developed a prototype measuring both vertical and horizontal magnetic fields with frequencies ranging from $800 \mathrm{kHz}$ to more than $20 \mathrm{MHz}$. In the two field surveys they mention, they use inter-coil spacings of 1,2 , and $4 \mathrm{~m}$ in order to determine the electrical properties of the first $5 \mathrm{~m}$ of soil. The use of these various spacings raises some important issues about assuring an accurate loop orientation in the field, leading to the increase of uncertainty in measurements. This issue, added to the temperature and electrical cable drifts, decrease the accuracy of the primary magnetic field. Normalized fields can't be calculated; instead the tilt angle and ellipticity of the magnetic field polarization ellipse are used to determine the electrical parameters. Moreover, if their results are promising, Stewart et al. (1994) encountered some technological limitations for: (i) spacing larger than $3 \mathrm{~m}$, leading to low signal strength, (ii) frequencies higher than $20 \mathrm{MHz}$, and (iii) the inversion process that 
required 20 hours of computation for 18 measurement stations. Bourgeois and Lenain (2002) propose a similar approach with spacings ranging from 2 to $32 \mathrm{~m}$ and frequencies that can be chosen between $391 \mathrm{kHz}$ and $12.5 \mathrm{MHz}$. If the signal strength in the higher frequency range and the speed of the inversion program has been improved, the issues regarding the loop orientation in the field and the measurement speed are still real.

We propose a novel approach using a fixed Slingram geometry allowing a better control of the mechanical strength and the loop relative orientations. Instead of focusing on 1D sounding, we want to develop a method of mapping the electrical conductivity and the dielectric permittivity of the first meters of the soil in a time efficient way. After a description of the prototype's characteristics, we present two different case studies with different water contents: sandy alluvia and clay loam.

For the frequency ranges, we adopt the International Telecommunication Union radio regulation rules: Low Frequencies (LF) for $30 \mathrm{kHz}<f<300 \mathrm{kHz}$, Medium Frequencies (MF) for $300 \mathrm{kHz}<f<3 \mathrm{MHz}$ and High Frequencies (HF) for $3 \mathrm{MHz}<f<30 \mathrm{MHz}$.

\section{Definition of the prototype's characteristics}

We choose to adapt the Slingram configuration technology, using a transmitter and a receiver coil, originally developed in the LF range, for the MF range. In fact, another development path would be to design an electrical field sensor, but this type of sensor is too sensitive to changes in its elevation. To reduce this disagreement the use of electrodes stuck in the ground is necessary. This practice will greatly decrease the measurement speed and the production of maps at a field scale would be thus limited. Moreover, our laboratory acquired lots of feedback in the low frequency range, developing prototypes using Slingram geometry.

Since the secondary fields measured in the LF domain are smaller than those expected in the MF range, the mechanical design conditions are much more drastic in the LF frequency range. 
122 Our knowledge in the development of EMI devices should ensure the construction of a mechanically robust and well-adapted instrument.

Different characteristics of the Slingram configuration can be chosen not only to adjust the investigation depth of the prototype, but also to ensure its ability to measure both electrical parameters. Modeling of a homogeneous ground and a layered ground with varying electrical parameters were performed using the forward modeling schemes developed by Tabbagh (1994).

The goal of modeling is to evaluate the value of the secondary magnetic field $\overrightarrow{H_{S}}$ created by the ground, normalized by the primary field $\overrightarrow{H_{P}}$ emitted by the transmitter coil $\mathrm{T}_{\mathrm{x}}$. Tabbagh (1994) showed that the influence of the magnetic permeability $\mu$ is weak in the medium frequency range; its value is thus taken to be equal to the magnetic permeability of free space $\left(\mu=\mu_{0}=4 \pi \cdot 10^{-7} \mathrm{H} / \mathrm{m}\right)$. The primary magnetic field in the air is determined using the quasi-static approximation. Indeed, at $10 \mathrm{MH}$ and for a $1 \mathrm{~m}$ inter-coil spacing, the difference between the total primary field and the quasi-static primary field is equal to $2 \%$ or less. For a moment of the transmitter coil equal to 1 , the primary field is thus equal to

$$
H_{p}=1 /\left(4 \pi r^{3}\right)
$$

For the determination of the secondary field, in this frequency range, the displacement currents occurring not only in the ground, but also in the air must be taken into account (Bourgeois and Lenain, 2002). The air is considered a dielectric infinite half space with a dielectric permittivity equal to the free space dielectric permittivity $\left(\varepsilon=\varepsilon_{0}=8.85 .10^{-12} \mathrm{~F} /\right.$ $m$ ) and an electrical conductivity equal to $0(\sigma=0 \mathrm{~S} / \mathrm{m})$. Moreover, as the transmitter coil is taken for a magnetic dipole, the Schelkunoff electrical potential vector $\vec{F}$ is introduced so that the electrical field $\vec{E}$ is expressed by $\vec{E}=-\overrightarrow{r o t}(\vec{F})$ :

(i) in the air, the secondary electrical potential $\overrightarrow{F_{S}}$ satisfies the equation: 


$$
\Delta \overrightarrow{F_{s}}+\varepsilon_{0} \mu_{0} \omega \cdot \overrightarrow{F_{s}}=0
$$

(ii) in the ground, the total electrical potential $\vec{F}$ satisfies the equation:

$$
\Delta \vec{F}-i \sigma \mu \omega \cdot \vec{F}+\varepsilon \mu \omega^{2} \cdot \vec{F}=0
$$

The magnetic field can thus be expressed as a function of the electrical potential $\vec{F}$ :

$$
\vec{H}=(1 / i \omega \mu) \cdot \overrightarrow{\operatorname{rot}}(\overrightarrow{\operatorname{rot}}(\vec{F}))
$$

In the PERP configuration, using the Hankel transform, the ratio between the secondary and the primary magnetic field is equal to:

$$
\frac{H_{s}}{H_{p}}=-r^{3} \cdot \int_{0}^{+\infty} e^{-2 u_{0} h} \frac{\lambda^{3}}{u_{0}} R(\lambda) \cdot J_{0}(\lambda r) d \lambda
$$

where $J_{0}$ is the Bessel function of the first kind of order $0 ; h$ is the height between the ground surface and the coil center; $r$ is the inter-coil spacing; $\lambda$ is the integration spatial frequency; $u$ and $u_{0}$ are two variables depending on $\lambda$ and on the complex wave number $k\left(u=\sqrt{\lambda^{2}-k^{2}}\right.$ and $u_{0}=\sqrt{\lambda^{2}-k_{0}{ }^{2}}$ with $k^{2}=-i \sigma \mu \omega+\varepsilon \mu \omega^{2}$ and $\left.k_{0}{ }^{2}=\varepsilon_{0} \mu_{0} \omega^{2}\right)$ and $R(\lambda)=\frac{u_{0}-u}{u_{0}+u}$.

\section{Choice of the Slingram configuration}

We chose to adopt a PERP configuration with a transmitter $T_{x}$ and a receiver $R_{x}$ taken respectively as an horizontal magnetic dipole and a vertical magnetic dipole (Figure 1). This configuration is, for 1D terrain, theoretically strictly equivalent to the one having a transmitter with a vertical axis and a receiver with a horizontal axis but, with this choice, the influence of the electromagnetic fields created by the surrounding LF or MF transmitters are reduced and the signal to noise ratio is thus improved. The prototype is named CE120: C for conductivity; E for epsilon (the Greek character symbolizing the dielectric permittivity); 120 for $1.20 \mathrm{~m}$ (the 
171 inter-coil spacing $\mathrm{T}_{\mathrm{x}}-\mathrm{R}_{\mathrm{x}}$ ). The first in situ measurements were performed using a $1.56 \mathrm{MHz}$

172 working frequency, but it is possible to reach lower and higher frequencies with the same 173 coils. The height of the device above the ground is equal to $0.1 \mathrm{~m}$.

174

The response of this chosen configuration to electrical resistivity and dielectric permittivity variations is presented in Figure 2 for a homogeneous ground. For a fixed 40 relative dielectric permittivity value, the response of the CE120 is determined with electrical resistivities ranging from 1 to $10^{4} \Omega \mathrm{m}$. For a fixed $50 \Omega \mathrm{m}$ electrical resistivity value, the response of the CE120 is determined for dielectric permittivities comprised between 1 and 1000. These electrical resistivity and dielectric permittivity values are based on values measured from different soil types in laboratory tests (Smith-Rose, 1933; Scott et al., 1967; Kutrubes, 1986; Olhoeft, 1987; Knoll and Knight, 1994).

Noise sources must be evaluated in order to determine a detection threshold for the measurements (Kessouri, 2012). Besides the ambient electromagnetic noise, the device itself is a source of noise. Indeed the electronic components and the coaxial cables create a first electromagnetic noise. The geometric strains create a second one. Taking into account these noise sources and after a series of tests, a detection threshold of the ratio $\mathrm{H}_{\mathrm{s}} / \mathrm{H}_{\mathrm{p}}$ equal to 100ppm can be adopted.

In Figure 2, we can see that the chosen configuration allows the simultaneous measurements of the electrical conductivity $\sigma$ (or the electrical resistivity $\rho=1 / \sigma$ ) and the relative dielectric permittivity $\varepsilon_{\mathrm{r}}$, for $\rho \leq 2185 \Omega \mathrm{m}$ (with a homogeneous relative dielectric permittivity fixed to 40 ) and for $\varepsilon_{\mathrm{r}} \geq 2.6$ (with a fixed homogeneous electrical resistivity equal to $50 \Omega \mathrm{m}$ ). These threshold values are still valid for higher fixed soil resistivities such as 500 , 1000 or $5000 \Omega \mathrm{m}$. These determined ranges of measurable properties fit the expected ranges of soil electrical conductivities and dielectric permittivities in the medium frequency range. 
The adopted configuration is thus well suited for the simultaneous measurements of the electrical conductivity and the dielectric permittivity at $1.56 \mathrm{MHz}$.

\section{Influence of the investigation depth}

We want to design a device that measures the apparent electrical conductivity and dielectric permittivity of the shallow subsurface, for a volume of soil being at least $1 \mathrm{~m}$ thick. To check the investigation depth that can be reached with the chosen characteristics of the CE120, we test its ability to detect a thin moving layer. The investigation depth represents the depth until which this thin layer can be detected. The former modeling equations are now applied to a 3 layered ground where the electrical conductivity and the dielectric permittivity of the upper and bottom layers are identical: $\rho_{1}=\rho_{3}=50 \Omega \mathrm{m} ; \varepsilon_{1}=\varepsilon_{3}=40$. The electrical resistivity of the second thin layer is fixed to $5 \Omega \mathrm{m}$ for figures $3 \mathrm{a}$ and $3 \mathrm{~b}$, and to $500 \Omega \mathrm{m}$ for figure $3 \mathrm{c}$ and $3 \mathrm{~d}$. The relative dielectric permittivity can be equal either to 5,50 , or 100 . The thickness of the second layer is fixed to $0.1 \mathrm{~m}$ for both modeling. Taking into account a detection threshold equal to 100ppm, the thin conductive layer (Figures $3 \mathrm{a}$ and $3 \mathrm{~b}$ ) can be detected until a $2.6 \mathrm{~m}$ depth. The investigation depth is significantly lower for the thin resistive layer (Figures 3c and 3d): with the same detection threshold, the investigation depth is now equal to $2 \mathrm{~m}$. Even though it is lower than for a conductive target, this investigation depth is important compared to those reached by LF slingram devices. Indeed, it is well known that the electromagnetic devices are not very sensitive to resistive features. This lack of sensitivity is even emphasized when the measurement's frequency is becoming lower. With our prototype, we are able to detect a thin resistive layer until $2 \mathrm{~m}$. This result is promising in terms of detection of resistive targets using medium frequencies.

The chosen properties for our prototype looks optimal not only in terms of range of values detected, both for the electrical conductivity and the dielectric permittivity, but also in 
221

222

terms of reached investigation depth, which is close to $2 \mathrm{~m}$, even in the worst cases. The CE120 prototype (Figure 4) was built using this specific configuration. The rigid box that contains the transmitter and the receiver coils is fixed on a three-wheel trolley that is electrically non-conductive and made with polyethylene. This configuration permits a fast measurement rate. After a calibration stage, the CE120 was tested at a field scale.

\section{Determination of the electrical parameters}

In order to map the electrical parameters of the soil, a calibration step of the prototype is necessary. Indeed, two steps are needed to transform the raw measurements into electrical conductivity and dielectric permittivity data: (i) a calibration step allowing to transform the raw data, expressed in an arbitrary electronic unit (digit), into real and imaginary parts of the magnetic field (in ppm), using a calibration coefficient (in ppm/digit); and (ii) an inversion scheme transforming these magnetic fields (expressed in ppm) into electrical parameters.

For the calibration process, explained in detail in Thiesson et al. (2014), we measure the response of the CE120 to a small conductive sphere and compare it to the expected theoretical variations. A calibration coefficient is obtained from this comparison. Moreover, to double check this result and to determine the zeros of the prototype (the offset variations that are mainly caused by the internal electrical noises of the device), a second experimentation is led, where the response of the CE120 for different elevations is measured. The theoretical response of the prototype is calculated using an electrical sounding at the same location. The comparison of the theoretical and measured responses of the CE120 for different elevations then allows for a check of the calibration coefficient and for a calculation the offset occurring both in the in phase and quadrature components.

At these frequencies, the electrical parameters are both influencing the real and imaginary parts of the magnetic fields. If, in the low frequency range, simple linear 
relationships exist between the magnetic fields and the electrical parameters, in the medium frequency range, an inversion procedure is needed to transform the real and imaginary parts of the magnetic fields into electrical parameters. The relationships can be found numerically by solving an inverse problem using the classical Newton-Raphson procedure or abacus (Thiesson et al., 2014).

\section{Field case studies}

\section{Objectives}

The prototype has been tested on two different soil types: sandy alluvia and clay loam. The objective was to determine the ability of the CE120 to detect water content variations first in a clay-free context, then in a clay-rich environment. Indeed, the water and clay content of soils are two of the main state properties governing the electrical parameters in the medium frequency range: they are mostly responsible for polarization mechanisms observed in the MF range. The volumetric water content of soil can be directly linked with the dielectric permittivity in the high frequency range and its role persists in the medium frequency range. The physical explanation of this major effect is the following: water molecules are dipolar molecules, possessing permanent electrical momentums; the application of an electrical field makes them rotate, creating a dipolar polarization.

In addition to this high frequency polarization effect, polarization processes are occurring at the interface between the different components of the porous medium between a few $\mathrm{kHz}$ and a few MHz. These mechanisms can be macroscopically brought together as Maxwell-Wagner effects or interfacial polarizations (Chen and Or, 2006; Leroy et al. 2008; Tabbagh et al., 2009). Two main mechanisms corresponding to Maxwell-Wagner effects can be observed in the medium frequency range. In presence of an electrical field, cations and ions are moving in opposite directions in the electrolyte until reaching interfaces between the 
solid grains and the electrolyte. An accumulation of positive charges on one side of the interfaces and negative charges on the other side can then be observed. Moreover, in presence of charged particles at the grain surfaces, the ions repartition in the electrical double layer changes and an electrochemical interfacial polarization can be observed. The measured dielectric permittivity is thus influenced by the nature of the charged particles, particularly their specific surface area and their cation-exchange capacity (CEC). The presence of clays, possessing a high specific surface area and a large CEC, play an important role and can significantly increase the value of the dielectric permittivity in the medium frequency range.

\section{Measurements on sandy alluvia}

A $20 \times 8 \mathrm{~m}$ plot of Quaternary sandy alluvia at the INRA d'Orléans (France) was chosen to explore the measured answer on a clay-free soil. A water content contrast was created artificially: the center of the plot was covered up using a $6 \times 8 \mathrm{~m}$ canvas sheet during 6 months before any measurement was made. The measurements were made during the dry period (in May) and half of the plot was sprinkled during 6 hours prior to the recordings. Three different zones, corresponding to three different water contents, were created that way. The measurements were taken every $1 \mathrm{~m}$, leading to 160 measurement points on the plot.

Besides measurements with the prototype, the electrical conductivity was also evaluated using a resistivity-meter (RMCA-4 from CNRS) and a pole-pole array (Figure 5) with a $1 \times 1 \mathrm{~m}^{2}$ mesh. The apparent electrical resistivity values are very high, ranging from $650 \Omega \mathrm{m}$ to more than $10000 \Omega \mathrm{m}$. Three areas can be clearly identified: (i) the covered-up area where the resistivities are lower (from $650 \Omega \mathrm{m}$ in the middle to $2410 \Omega \mathrm{m}$ at the boundaries); (ii) the resistivities of the watered area are ranging between 2100 and $4650 \Omega$ m; (iii) the most electrically resistant area is found on the south-eastern part of the plot where the electrical resistivities reach values above $10000 \Omega \mathrm{m}$ for the bare soil. We used the same scale frame to 
represent electrical resistivities measured by the CE120 prototype (Figure 6). The values obtained are slightly lower than those measured using the resistivity-meter. The three different areas cannot be differentiated, but the boundaries of the covered up area are marked by lower resistivity zones. Looking at the resistivity values, it is clear that we reached the boundary of the noise level of the EM prototype. These observations are in good agreement with the previous modeling (Figures 2 and 3) where the sensitivity limit of the prototype to the electrical resistivity was determined equal to $2185 \Omega \mathrm{m}$ (for a homogeneous ground with $\varepsilon_{\mathrm{r}}=$ 40). Even if we reached the sensitivity limits, the lateral boundaries between the three different moisture areas are detected, which indicate a significant sensitivity of the CE120 to the boundaries between areas with changing electrical resistivities.

The apparent dielectric permittivity was also determined using the CE120 measurements (Figure 7). Three different areas, corresponding to the three different moisture contents can be identified: (i) the lower dielectric permittivities correspond to the bare soil area; (ii) in the middle of the plot, the higher dielectric permittivities are measured in the covered up area; and (iii) in the watered area, the dielectric permittivites are varying from 8 to 18.

An interesting artifact is observed in the covered up area, which can be divided into two distinct zones. In the north-west area, the values of the dielectric permittivity are higher than in the south-east area. This variation can be explained by the fact that the north-west area has been not only covered-up for 6 months, restricting the water evaporation, but also watered for 6 hours prior to measurements. This distinction is only visible on the dielectric permittivity map and is not detected by the DC resistivity measurements. The dielectric permittivity is thus an interesting parameter bringing along new information. 
The values, ranging from 1.5 to 19.5 , are close to those expected for sands in the high frequency range. The interfacial polarization processes (Maxwell-Wagner effects) expected in the MF range don't seem to occur in this clay-free context. Consequently we can apply the Topp et al. equation (Topp et al., 1980) to the relative dielectric permittivities $\varepsilon_{r}$ in order to deduce the volumetric water content $\theta_{v}$ :

$$
\theta_{v}=-5 \cdot 3 \cdot 10^{-2}+2 \cdot 92 \cdot 10^{-2} \cdot \varepsilon_{r}-5 \cdot 5 \cdot 10^{-4} \cdot \varepsilon_{r}{ }^{2}+4 \cdot 3 \cdot 10^{-6} \cdot \varepsilon_{r}{ }^{3}
$$

The obtained volumetric water content map can be compared to mass water content measurements of soil samples (Figure 8). The samples were taken from the surface to a depth of $60 \mathrm{~cm}$, every $10 \mathrm{~cm}$. Because of the hardness of the soil, we were not able to dig deeper with the auger. The values of mass water content are consistent with the expectations: they are lower (between 3 and 4\%) in the bare soil area, constant and close to $7.5 \%$ in the covered up area, and decrease from $10-15 \%$ to $6-7 \%$ as we go deeper for the watered area. If we compare the calculated volumetric water content and the measured mass water content at the sounding point, we obtain a mean apparent dry density equal to $1.3 \mathrm{~g} / \mathrm{cm}^{3}$. Indeed, the relation between the two water contents is equal to:

$$
w=\theta_{v} \cdot \frac{\rho_{w}}{\rho_{a s}}
$$

Where $w$ is the mass water content over the dry specific mass; $\theta_{v}$ is the volumetric water content; $\rho_{w}$ is the water density and $\rho_{a s}$ is the apparent dry density.

The calculated mean apparent dry density is in good agreement with apparent dry densities found for sandy soils (Donahue et al., 1977). In a clay-free soil, the volumetric water content can be calculated applying classical high frequency relations like Topp et al. equation to the dielectric permittivitiies measured with the CE120 at $1.56 \mathrm{MHz}$.

\section{Measurements on a clay-loam soil}


In order to test the influence of clay on the CE120 measurements, we performed a survey on a clay-loam soil at the ORE ACBB from INRA Estrée-Mons (France). Water content variations on the plot were generated by the crop growth. Indeed, the $8 \times 3 \mathrm{~m}$ plot was set at the border between the bare soil and the soil covered by wheat. Measurements were taken in March and in May 2011. In March, the wheat had just been planted and no water contrast was expected between the two areas, but in May, the wheat root network had developed and reached $1 \mathrm{~m}$ depth, creating important water contrasts between the wheat cover and the bare soil.

These contrasts can be observed in the DC electrical conductivity measurements (Figure 9). Two different devices were used, depending on their availability: the RM15-D from Geoscan Research and the RMCA-4 (CNRS). Pole-pole measurements were performed with the RM15-D in March on a 0.5m grid mesh, allowing three different electrode spacing $(0.5 \mathrm{~m}, 1 \mathrm{~m}$ and $1.5 \mathrm{~m})$. The RMCA-4 resistivity-meter was used in May with a Wenner $\alpha$ configuration and a $1 \mathrm{~m}$ electrode spacing. Looking at the $1 \mathrm{~m}$ electrode spacing in May (Figure $9 \mathrm{~d}$ ), a clear difference is observed between the western part of the plot, covered with wheat and the eastern part: the electrical conductivity is lower on the wheat cover. Moreover, the values of the bare soil in May are close to those of the entire plot in March. These observations confirm the influence of the wheat grow on the soil water content, and thus on the DC electrical conductivity measurements.

The same variations can be observed on the electrical conductivity map calculated with the CE120. However, the electrical resistivity values are higher than those measured for the DC electrical resistivity and the contrast between the bare soil and the wheat cover in May is weaker.

The dielectric permittivity map shows the same patterns. The values are generally higher in March than in May. The bare soil of May has lower values, but in the same order of 
magnitude than in March (around 80-90). The influence of the wheat growing is clearly visible in May: the western part of the plot has lower values of permittivities (between 40 and 69). Compared to the high frequency relative dielectric permittivities, ranging from 1 (for vacuum) to 81 (for pure water) in soils, the measured values seem high. Yet, in a clay-rich context, where the amount of clay is reaching $20 \%$, these values can be expected at $1.56 \mathrm{MHz}$. To confirm these results, laboratory measurements, using a capacitive cell coupled with a frequency response analyzer (Kessouri, 2012), were made on samples collected on site (Figure 11). The relative dielectric permittivity of the samples was evaluated at $1.024 \mathrm{MHz}$ and $2.048 \mathrm{MHz}$ for different volumetric water contents. Depending on the water content, the dielectric permittivity ranges from 3.6 to 367.3 , respectively for a dry and saturated sample. The values obtained in situ are coherent with these measurements. An empirical relationship between the volumetric water content and the relative dielectric permittivity can be deduced from the laboratory measurements with a coefficient of determination $\mathrm{R}^{2}$ equal to 0.778 for 47 data points:

$$
\theta=0.40\left(1-e^{-\varepsilon_{r} / 62.6}\right)
$$

This expression is used to determine the apparent volumetric water content of the soil in situ (Figure 12). In March 2011, the apparent volumetric water content is fairly homogeneous and equal to $31 \%$ in average.

Measurements of the mass water content were also performed on two different locations (P1 on the wheat cover and P2 on the bare soil) of the plot (Figure 12a and 12b). The mass water content versus depth appears to be constant and equal to $21 \%$. Using these values of volumetric and mass water content in equation (7), we found an apparent dry density equal to 1.5 . We used this calculated apparent dry density to evaluate the mass water content of the soil in May 2011, using the dielectric permittivity measurements. In May, the volumetric water contents are equal, in mean, to $31 \%$ for the bare soil and to $22 \%$ for the 
wheat cover. Using the dry apparent density found for the measurements in March, we obtain mass water contents equal to $20.7 \%$ for the bare soil and $14.7 \%$ for the wheat cover. These values are coherent with the mean values of the mass water content measurement over depth.

evaluate the volumetric water content variations of a clay rich soil with an investigation depth overpassing $2 \mathrm{~m}$. Since there is a lack of models regarding the relationship between the MF dielectric permittivity and the water content of soils, the laboratory measurements are a necessary step to find a "calibration" equation between these two properties for a given soil and it has to be done for each new soil type.

\section{Conclusion}

In order to estimate simultaneously the electrical conductivity and the dielectric permittivity of soils, we developed a new EM prototype working in the medium frequency range. Its investigation depth is higher than 2 meters, especially in clay rich context where the GPR imaging is considerably attenuated. The CE120 is using a PERP Slingram geometry with a fixed inter-coil spacing of $1.2 \mathrm{~m}$ and a working frequency of $1.56 \mathrm{MHz}$. If the inter-coil spacing is fixed to reduce the geometric noise source, the same coil configuration allows for measurements at various frequencies in the medium frequency range.

Analytical modeling enables us to check the properties of the prototype: (1) the simultaneous measurement of $\sigma$ and $\varepsilon_{\mathrm{r}}$ is possible for electrical resistivities lower than $2185 \Omega \mathrm{m}$, and for dielectric permittivities higher than 2.6, which covers the whole range of encountered values in the medium frequency range for soils; and (2) the investigation depth of the CE120 is reaching $3 \mathrm{~m}$ for conductive targets and becomes close to $2 \mathrm{~m}$ for resistive 
targets. It thus represents a good compromise between investigation depth and lateral resolution.

To reach the electrical conductivity and the dielectric permittivity using the CE120 measurements, two steps are necessary: (1) a calibration step where the calibration coefficient is calculated by comparing modeling to measurements for a known object and/or for a height variation; and (2) an inversion step where the electrical parameters are deduced from the magnetic field ratio with the Newton-Raphson procedure.

Two different field test sites were chosen according to their clay and water content variations in order to study the prototype's response to water variations for a clay-free and a clay-rich soil.

For the sandy alluvia, located in the INRA of Orleans, the clay content is close to 0 and the water content variations were artificially created. The resistivities measured by the DC resistivity-meter are mostly higher than the resistivities detected by the CE120. The prototype is thus unable to measure properly the soil electrical conductivity. Still, the boundaries between the wet and dry part of the plot are clearly visible. As the LF-EM methods are usually not adapted to very resistive context, this result is encouraging regarding the use of medium frequency EM devices in electrically resistive environments. Moreover, the measured dielectric permittivity has been successfully used to estimate the volumetric water content of the soil using the HF equation of Topp et al. (1980). Indeed, on this resistive kind of soil, the Maxwell-Wagner effects are low and only the polarization of the water molecules is observable. These measurements are all the more consistent that the comparison between the calculated volumetric water content and the measured mass water content is convincing.

On the clay loam, the soil state parameters variations are detected not only by $\varepsilon_{\mathrm{r}}$, but also by $\sigma$, even though the variations are higher for the relative dielectric permittivity. The 
measured values of $\varepsilon_{\mathrm{r}}$ are high (close from 100), showing the presence of the MaxwellWagner polarization effects at $1.56 \mathrm{MHz}$. With an amount of clay close to $20 \%$, these values can be expected in the medium frequency range. Thus, the Topp et al. equation can no longer be used to calculate the soil volumetric water content. As no general relationships between $\theta_{\mathrm{v}}$ and $\varepsilon_{\mathrm{r}}$ are known in the medium frequency range, a different expression needs to be found for each case study. We determined an empirical relationship from a series of laboratory measurements on the same soil type. The volumetric water contents obtained are coherent with the mass water content measured on site.

The CE120 is thus adapted for shallow investigations of water content variations, as well as absolute value estimations, both in electrically conductive and none conductive contexts. One of the restraints of this estimation is the necessity of finding a "calibration" equation linking the dielectric permittivity and the water content for each type of soil. Indeed, laboratory measurements for each survey can be time consuming. Some work needs to be done to explore the relationships between the electrical parameters and the water content of soils in the medium frequency range, both theoretically and experimentally. Moreover, as the dielectric permittivity and electrical conductivity are both frequency dependent, information can be added to the measurements by adding several frequencies to the prototype's available working frequencies. The same coils and geometry can be used for the whole medium frequency range. Measuring the electrical parameters at different frequencies also includes taking into account the dispersive character of the parameters in the model schemes. This is not easy as there is no model describing the Maxwell-Wagner polarization processes as a function of the state properties of the ground. The medium frequency range thus offers an interesting path to study the frequency dependence of the electrical parameters and their relations with the soil state properties. 
We would like to thank the INRA d'Orléans, particularly Isabelle Cousin and Maud

470 Séger, not only for letting us prospect one of their plot, but also for their availability and their

471 help. Our gratitude also goes to the INRA d'Estrée-Mons and the ORE-ACBB, particularly to

472 Nicolas Brunet and Hubert Boisard for their authorization, their support and their advice 473 while we were prospecting their plots.

474 


\section{REFERENCES}

476

477 Bourgeois, B., and Lenain, F., 2002, Etude de la faisabilité d'un appareil électromagnétique 478 fréquentiel dans la gamme $30 \mathrm{kHz}-10 \mathrm{MHz}$, rapport BRGM RC-51643-FR.

479 Chen, Y., and Or, D., 2006, Effects of Maxwell-Wagner polarization on soil complex 480 dielectric permittivity under variable temperature and electrical conductivity: Water Resources Research, 42, W06424.

Donahue, R. L., Miller R. W., and Shickluna, J. C., 1977, Soils: An Introduction to Soils and Plant Growth: Prentice-Hall, 626p. ISBN 0-13-821918-4.

Friedman, S. P., 2005, Soil properties influencing apparent electrical conductivity: a review: Computers and Electronics in Agriculture, 46, 45-70.

Huang, H., and Fraser, C. D., 2002, Dielectric permittivity and resistivity mapping using highfrequency, helicopter-borne EM data: Geophysics, 67, no. 3, 727-738.

Kessouri P., 2012, Mesure simultanée aux fréquences moyennes et cartographie de la permittivité diélectrique et de la conductivité électrique du sol: $\mathrm{PhD}$ Thesis, Pierre et Marie Curie University, Paris, France.

Knight, R. J., 2001, Ground Penetrating Radar for Environmental Applications: Annual Review of Earth and Planetary Sciences, 29, 229-255.

Knoll, M. D., and Knight, R. J., 1994, Dielectric and hydrogeologic properties of sand-clay mixtures: Proceedings of the fifth international conference on Ground Penetrating Radar, Kitchner, Canada.

Kutrubes, D. L., 1986, Dielectric permittivity measurements of soils saturated with hazardous fluids: PhD Thesis, Colorado School of Mines, Golden, USA.

Leroy, P., Revil, A., Kemna, A., Cosenza, P., and Ghorbani, A., 2008, Complex conductivity of water saturated packs of glass beads: Journal of Colloid Interface Science, 321, 103-117. 
500

501

502

503

504

505

506

507

508

509

510

511

512

513

514

515

516

517

518

519

520

521

522

Liu, Q., Roberts, A.P., Larrasoaña, J.C., Banerjee, S.K., Guyodo, Y., Tauxe, L., and Oldfield, F., 2012, Environmental magnetism: Principles and applications: Review of Geophysics, 50, RG4002, doi:10.1029/2012RG000393.

Olhoeft, G. R., 1987, Electrical properties from $10^{-3}$ to $10^{9} \mathrm{~Hz}$ - physics and chemistry: Physics Chemistry of Porous Media II, American Institute of Physics Conference Proceeding, 154, 281-298.

Scott, J. J., Carroll, R. D., and Cunningham, D. R., 1967, Dielectric constant and electrical conductivity measurements of moist rock: a new laboratory method: Journal of Geophysical Research, 72, 5101-5115.

Smith-Rose, R. L., 1933, The electrical properties of soils for alternating currents at radio frequencies: Proceedings of the Royal Society A, 140, 359-377.

Stewart, D.C., Anderson, W.L., Grover, T.P., and Labson, V.F., 1994, Shallow subsurface mapping by electromagnetic sounding in the $300 \mathrm{kHz}$ to $30 \mathrm{MHz}$ range: Model studies and prototype system assessment: Geophysics, 59, no. 8, 1201-1210.

Tabbagh, A., 1994, Simultaneous Measurement of electrical conductivity and dielectric permittivity of soils using a Slingram electromagnetic device in medium frequency range: Archeometry, 36, no. 1, 159-170.

Tabbagh, A., Cosenza, P., Ghorbani, A., Guérin, R., and Florsch, N., 2009, Modelling of Maxwell-Wagner Induced Polarisation Amplitude for Clayey Materials: Journal of Applied Geophysics, 67, no. 2, 109-113.

Thiesson, J., Kessouri, P., Schamper, C., and Tabbagh, A., 2014, About calibration of frequency domain electromagnetic devices used in near surface surveying: Near Surface Geophysics, doi: 10.3997/1873-0604.2014012. 
523 Topp, G. C., Davis, J. L., and Annan, A. P., 1980, Electromagnetic determination of soil 524 water content: measurements in coaxial transmission lines: Water Resources Research, 16, no. $525 \quad 3,574-582$.

526 Walther, E. G., Pitchford, A. M., and Olhoeft, G. R., 1986, A strategy for detecting subsurface 527 organic contaminants: Proceedings of the National Water Well Association/API, Conference 528 on Petroleum Hydrocarbons and Organic Chemicals in Ground Water - prevention, detection 529 and restoration, Houston, 357-81.

530 
Figure 1: Representation of the measurement device using a PERP Slingram configuration

534 with the transmitter coil $\mathrm{T}_{\mathrm{x}}$ (of moment $\vec{M}$ ) in horizontal magnetic dipole configuration, creating a primary magnetic field $\overrightarrow{H_{p}}$ and the receiver coil $\mathrm{R}_{\mathrm{x}}$ measuring the vertical component $\overrightarrow{H_{z s}}$ of the secondary magnetic field $\overrightarrow{H_{s}}$ created by a target in the ground. $\overrightarrow{H_{t}}$, the total magnetic field, is the sum of the primary and the secondary magnetic field and $\overrightarrow{H_{z t}}$ is its vertical component.

Figure 2: Modeling of the real (for a) and c)) and imaginary (for b) and d)) parts of the ratio between the secondary magnetic field $H_{s}$ and the primary magnetic field $H_{p}\left(H_{p}=1 / 4 \pi r^{3}\right.$, with $r=1.20 \mathrm{~m}$ the inter-coil spacing) for a homogeneous ground. The coils, set in PERP

542 configuration, are placed at a height of $0.1 \mathrm{~m}$. The measurement frequency is fixed at 543 1.56MHz. The results are represented as a function of the electrical resistivity of the ground (for a) and b)) and the relative dielectric permittivity (for c) and d)). The reference characteristics of the homogeneous ground are $50 \Omega \mathrm{m}$ for the electrical resistivity, 40 for the 546 relative dielectric permittivity and $30.10^{-5} \mathrm{uSI}$ for the magnetic susceptibility. The striped area 547 corresponds to the area where the variations of the ratios are no longer detected if the 548 detection threshold is fixed to 100ppm.

549 Figure 3: Modeling of the real (for a) and c)) and imaginary (for b) and d)) parts of the ratio 550 between the secondary magnetic field $H_{s}$ and the primary magnetic field $H_{p}\left(H_{p}=1 / 4 \pi r^{3}\right.$, 551 with $r=1.20 \mathrm{~m}$ the inter-coil spacing) for a 3-layered ground. The coils, set in PERP 552 configuration, are placed at a height of $0.1 \mathrm{~m}$. The measurement frequency is fixed at $553 \quad 1.56 \mathrm{MHz}$. The results are represented as a function of the $1^{\text {st }}$-layer thickness $e_{1}$ for various 
555 parameters of the $1^{\text {st }}$ and $3^{\text {rd }}$ layers are equal to the reference characteristics of the 556 homogeneous ground $\left(\rho=50 \Omega m, \varepsilon_{r}=40, \kappa=30 \cdot 10^{-5} u S I\right)$. The thin mobile layer has a 557 thickness $e_{2}$ equal to $0.1 \mathrm{~m}$, a magnetic susceptibility of $30.10^{-5} u S I$, a relative dielectric 558 permittivity equal to either 5,50 or 100 , and an electrical resistivity equal to $5 \Omega m$ for the 559 conductive case (for a) and b)) or to $500 \Omega m$ for the resistive case (for c) and d)). The striped 560 area corresponds to the area where the variations of the ratios are no longer detected if the

561

562

563

564

565

566

567

568

569

570

571

572

573

574

575

576

577

578

detection threshold is fixed to 100ppm. The grey area corresponds to the detection threshold area around the medium ratio value when $e_{1}$ is significant.

Figure 4: Measurement device made up of the CE120 prototype and its trolley, under use on the clay-loam soil, at the threshold between the bare soil and the wheat cover (see Fig. 8 to 10).

Figure 5: Map of the electrical resistivity of sandy alluvia, measured with an RMCA-4 (CNRS) resistivimeter, using a Pole-Pole electrode configuration $(A M=1 \mathrm{~m})$ on a $1 \mathrm{~m}$ grid mesh. Artificial water content contrasts were created using a $6 \times 8 \mathrm{~m}$ canvas sheet during 6 months (covered up area) and a sprinkler during 6 hours (watered area). The plot is entirely laid to grass.

Figure 6: Map of the electrical conductivity of sandy alluvia, measured with the CE120 on a $1 \mathrm{~m}$ grid mesh. Artificial water content contrasts were created using a $6 \times 8 \mathrm{~m}$ canvas sheet during 6 months (covered up area) and a sprinkler during 6 hours (watered area). The plot is entirely laid to grass.

Figure 7: Map of the dielectric permittivity of sandy alluvia, measured with the CE120 on a $1 \mathrm{~m}$ grid mesh. Artificial water content contrasts were created using a $6 \times 8 \mathrm{~m}$ canvas sheet during 6 months (covered up area) and a sprinkler during 6 hours (watered area). The plot is entirely laid to grass. 
579 Figure 8: a) Map of the volumetric water content of sandy alluvia, determined using the 580 CE120 measurements and the Topp et al. equation (Topp et al., 1980) on a 1m grid mesh. 581 Artificial water content contrasts were created using a $6 \times 8 \mathrm{~m}$ canvas sheet during 6 months 582 (covered up area) and a sprinkler during 6 hours (watered area). The plot is entirely laid to 583 grass. b) Mass water content measured at the PiLj location points on the map a) as a function 584 of the studied sample depth.

585 Figure 9: Maps of the electrical resistivity of a clay-loam soil, measured with an RM15-D 586 (Geoscan Research) resistivimeter in March 2011 (for a), b) and c)), using a Pole-Pole 587 electrode configuration (a) $A M=0.5 \mathrm{~m}$; b) $A M=1 \mathrm{~m}$; c) $A M=1.5 \mathrm{~m}$ ) on a $0.5 \mathrm{~m}$ grid mesh and an RMCA-4 (CNRS) resistivity-meter in May 2011 (for d)), using a Wenner $\alpha$ electrode configuration $(a=1 \mathrm{~m})$ on a $0.5 \mathrm{~m}$ grid mesh. Wheat has been planted on the western half of the plot, while its eastern part is let bare.

Figure 10: Maps of the electrical conductivity (for a) and b)) and the dielectric permittivity 592 (for c) and d)) of a clay-loam soil, measured with the CE120 in March (for a) and c)) and May (for b) and d)) 2011 on a $0.5 \mathrm{~m}$ grid mesh. Wheat has been planted on the western half of the 594 plot, while its eastern part is let bare.

Figure 11: Laboratory measurements of the relative dielectric permittivity $\varepsilon_{\mathrm{r}}$ of soil samples from the plot of ORE-ACBB at INRA d'Estrée-Mons for various volumetric water contents $\theta$ $\left(\sigma_{\mathrm{w}}=536 \mu \mathrm{S} / \mathrm{cm} \pm 4 \%\right.$ at $\left.25^{\circ} \mathrm{C}\right)$. Measurements of the complex dielectric permittivity were made with a capacitive cell coupled to a frequency response analyzer at two different 599 frequencies $(1.024 \mathrm{MHz}$ and $2.048 \mathrm{MHz})$. A good fit was found for $\theta=0.40\left(1-\exp \left(-\varepsilon_{\mathrm{r}} / 62.6\right)\right)$ with a determination coefficient $\mathrm{R}^{2}$ of 0.779 for 47 data points. 
601 Figure 12: c) and d): Maps of the volumetric water content of the clay-loam soil, determined 602 using the CE120 measurements and laboratory experimentations (Kessouri, 2012) on a $0.5 \mathrm{~m}$ 603 grid mesh in March (for c)) and May (for d)) 2011. a) and b): Mass water content measured at 604 the Pi location points on the maps c) and d) as a function of the studied sample depth. The soil 605 textural characterization versus depth has been added to the graph.

606

607 


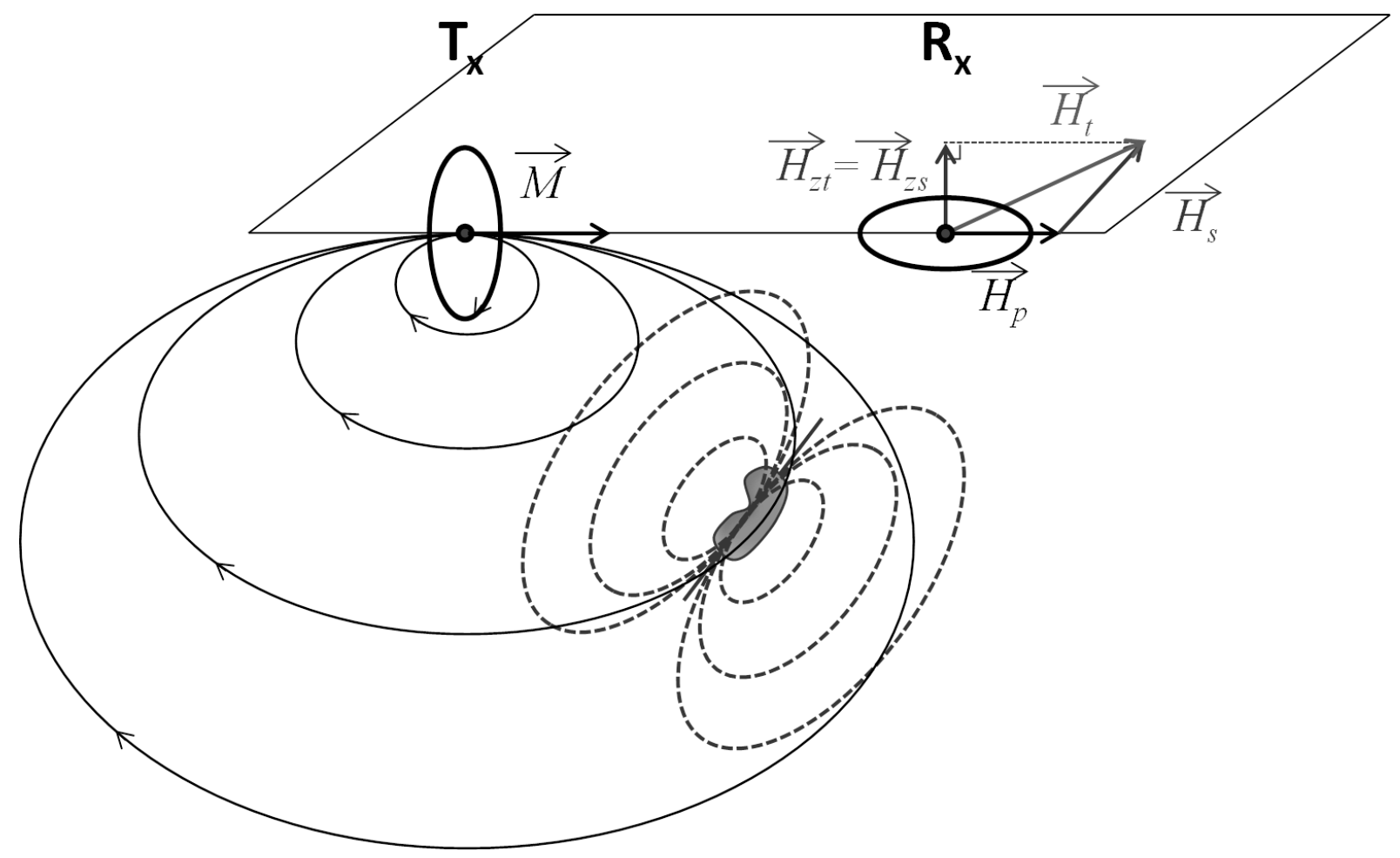

611 Figure 1: Representation of the measurement device using a PERP Slingram configuration with the 612 transmitter coil $\mathrm{T}_{\mathrm{x}}$ (of moment $\vec{M}$ ) in horizontal magnetic dipole configuration, creating a primary 613 magnetic field $\overrightarrow{H_{p}}$ and the receiver coil $\mathrm{R}_{\mathrm{x}}$ measuring the vertical component $\overrightarrow{H_{z S}}$ of the secondary 614 magnetic field $\overrightarrow{H_{s}}$ created by a target in the ground. $\overrightarrow{H_{t}}$, the total magnetic field, is the sum of the 615 primary and the secondary magnetic field and $\overrightarrow{H_{z t}}$ is its vertical component. 


\section{Homogeneous ground}

$$
\begin{aligned}
& \rho=50 \Omega . m \\
& \varepsilon_{r}=40
\end{aligned}
$$
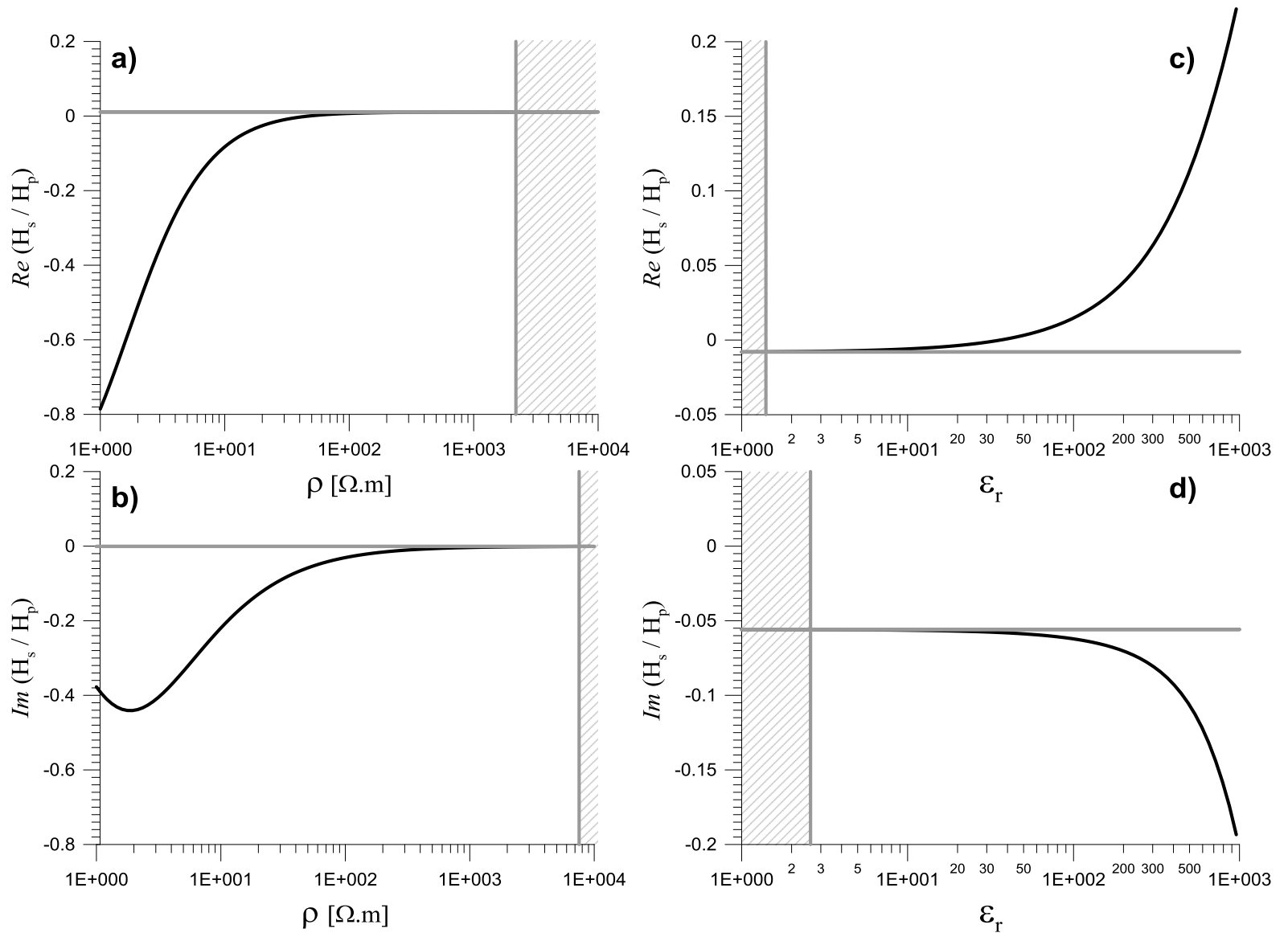

Figure 2: Modeling of the real (for $\mathbf{a}$ ) and $\mathbf{c}$ )) and imaginary (for $\mathbf{b})$ and $\mathbf{d}$ )) parts of the ratio between the secondary magnetic field $H_{s}$ and the primary magnetic field $H_{p}\left(H_{p}=1 / 4 \pi r^{3}\right.$, with $r=1.20 \mathrm{~m}$ the inter-coil spacing) for a homogeneous ground. The coils, set in PERP configuration, are placed at a height of $0.1 \mathrm{~m}$. The measurement frequency is fixed at $1.56 \mathrm{MHz}$. The results are represented as a function of the electrical resistivity of the ground (for a) and b)) and the relative dielectric permittivity (for $\mathbf{c}$ ) and d)). The reference characteristics of the homogeneous ground are $50 \Omega \mathrm{m}$ for the electrical resistivity, 40 for the relative dielectric permittivity and $30.10^{-5} \mathrm{uSI}$ for the magnetic susceptibility. The striped area corresponds to the area where the variations of the ratios are no longer detected if the detection threshold is fixed to $100 \mathrm{ppm}$. 

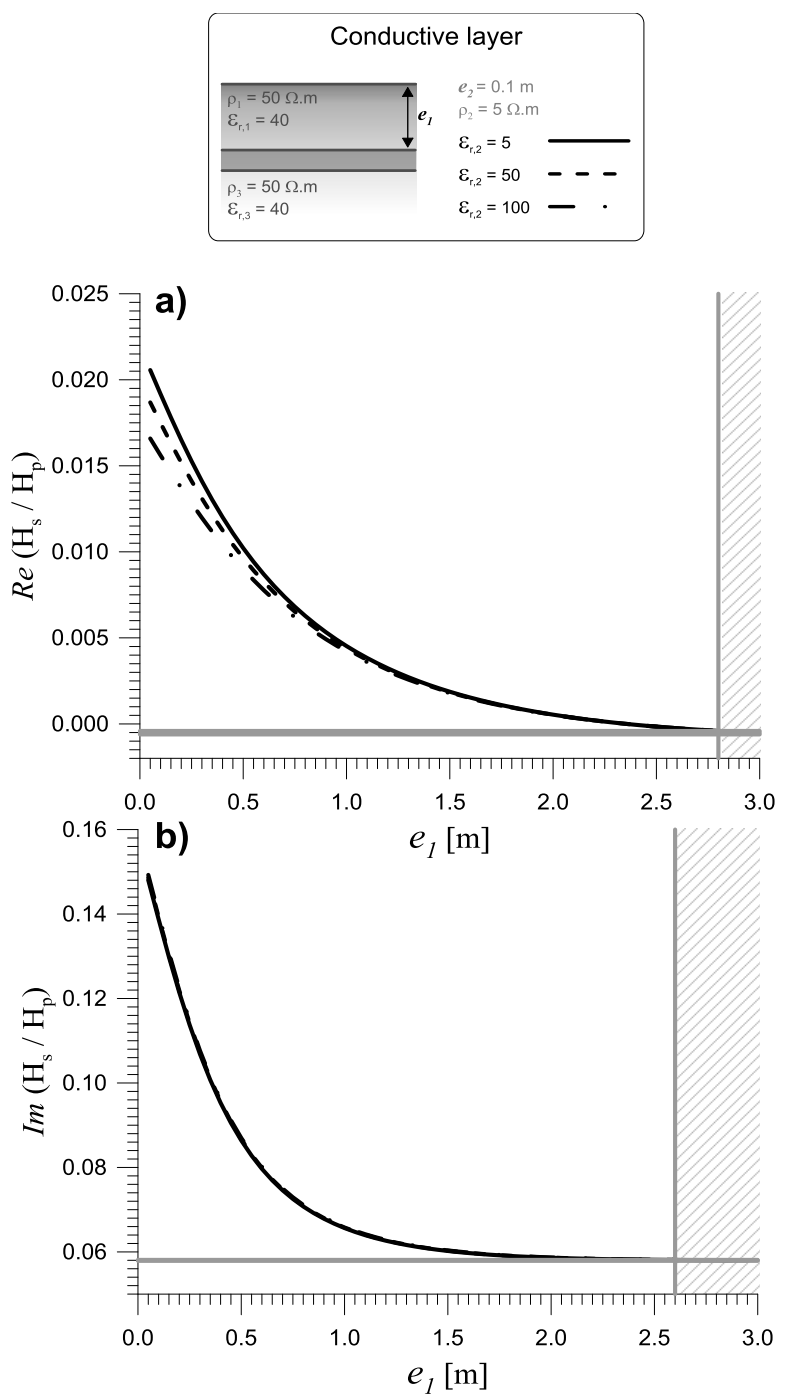
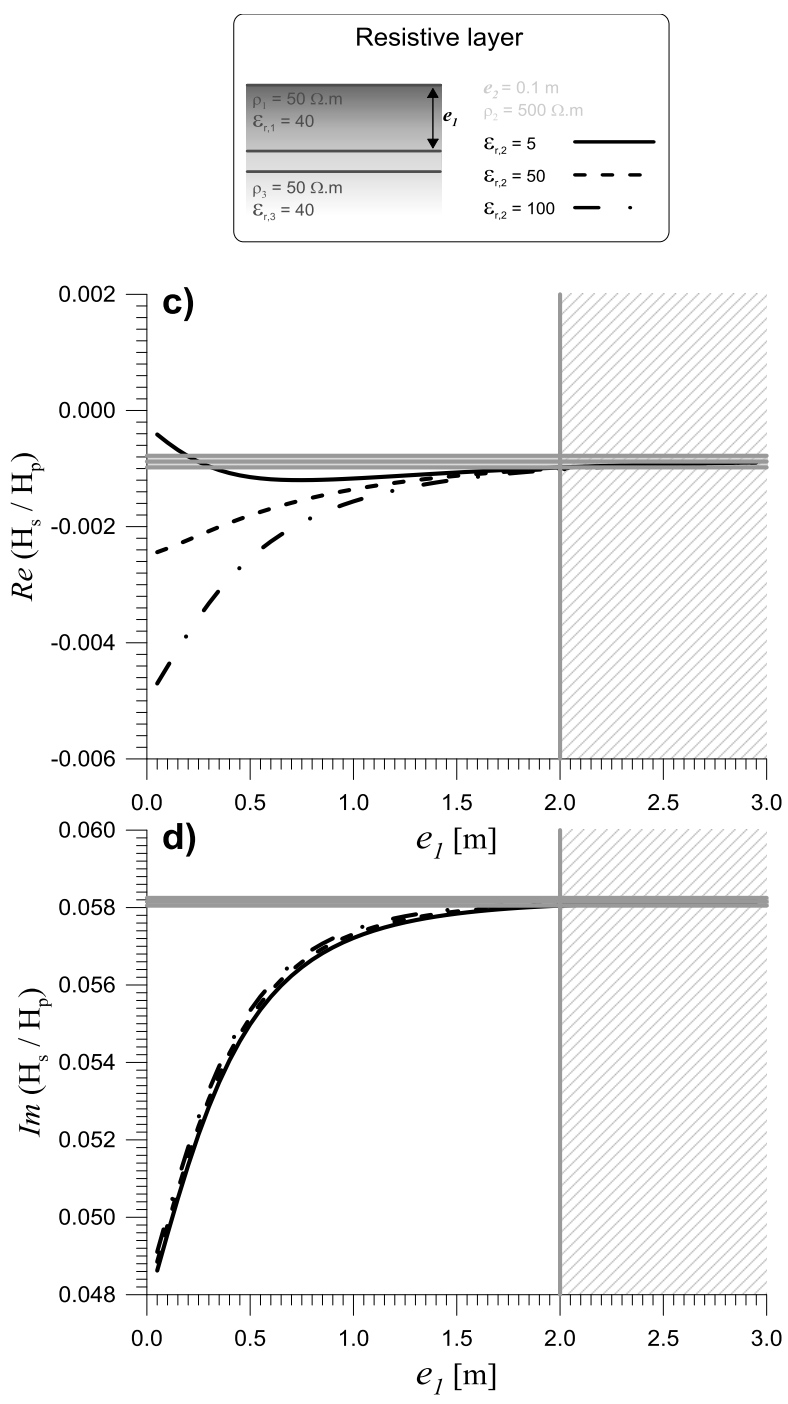

629

630

631

632

633

634

635

636

637

638

639

640

641

642

Figure 3: Modeling of the real (for $\mathbf{a}$ ) and $\mathbf{c}$ )) and imaginary (for $\mathbf{b}$ ) and d)) parts of the ratio between the secondary magnetic field $H_{s}$ and the primary magnetic field $H_{p}\left(H_{p}=1 / 4 \pi r^{3}\right.$, with $r=1.20 \mathrm{~m}$ the inter-coil spacing) for a 3-layered ground. The coils, set in PERP configuration, are placed at a height of $0.1 \mathrm{~m}$. The measurement frequency is fixed at $1.56 \mathrm{MHz}$. The results are represented as a function of the $1^{\text {st }}$-layer thickness $e_{1}$ for various electrical parameters of the $2^{\text {nd }}$ layer, considered as the thin mobile layer. The electrical parameters of the $1^{\text {st }}$ and $3^{\text {rd }}$ layers are equal to the reference characteristics of the homogeneous ground $\left(\rho=50 \Omega m, \varepsilon_{r}=40, \kappa=30.10^{-5} u S I\right)$. The thin mobile layer has a thickness $e_{2}$ equal to $0.1 \mathrm{~m}$, a magnetic susceptibility of $30.10^{-5} \mathrm{uSI}$, a relative dielectric permittivity equal to either 5,50 or 100 , and an electrical resistivity equal to $5 \Omega \mathrm{m}$ for the conductive case (for a) and b)) or to $500 \Omega m$ for the resistive case (for c) and d)). The striped area corresponds to the area where the variations of the ratios are no longer detected if the detection threshold is fixed to $100 \mathrm{ppm}$. The grey area corresponds to the detection threshold area around the medium ratio value when $e_{1}$ is significant. 


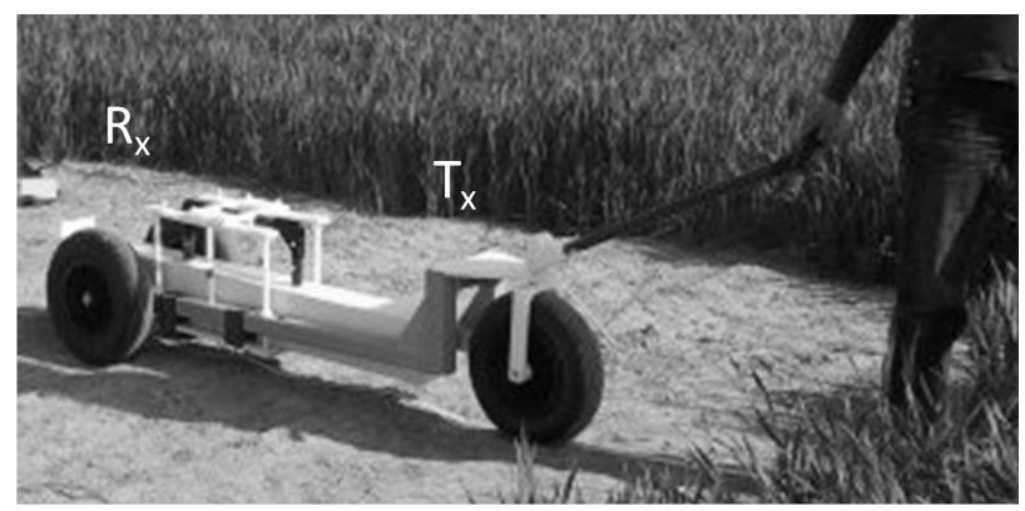

645 Figure 4: Measurement device made up of the CE120 prototype and its trolley, under use on the clay646 loam soil, at the threshold between the bare soil and the wheat cover (see Fig.8 to 10). 


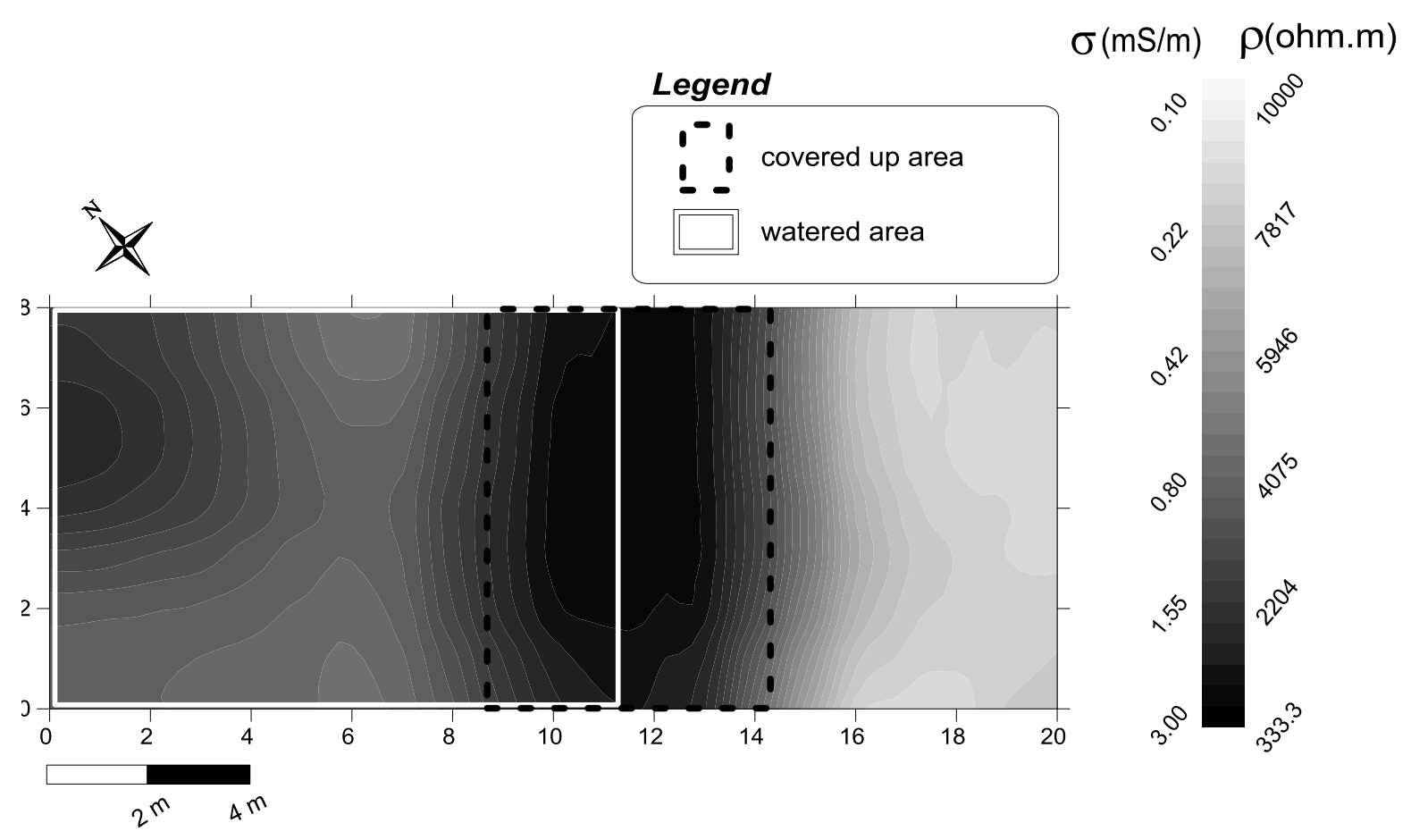

Figure 5: Map of the electrical resistivity of sandy alluvia, measured with an RMCA-4 (CNRS) resistivity-meter, using a Pole-Pole electrode configuration $(A M=1 \mathrm{~m})$ on a $1 \mathrm{~m}$ grid mesh. Artificial water content contrasts were created using a $6 \times 8 \mathrm{~m}$ canvas sheet during 6 months (covered up area) and a sprinkler during 6 hours (watered area). The plot is entirely laid to grass.

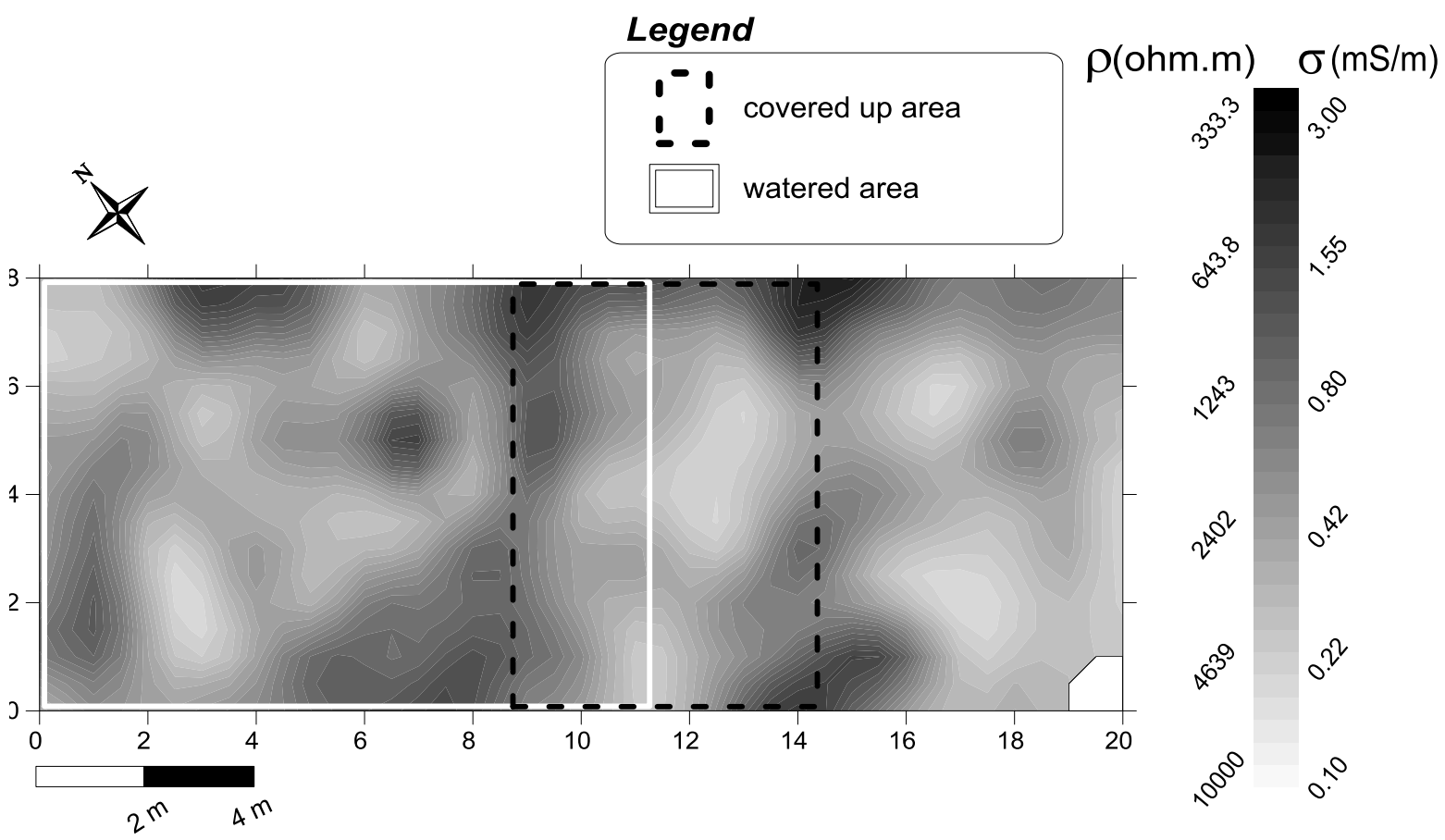

Figure 6: Map of the electrical conductivity of sandy alluvia, measured with the CE120 on a $1 \mathrm{~m}$ grid mesh. Artificial water content contrasts were created using a $6 \times 8 \mathrm{~m}$ canvas sheet during 6 months (covered up area) and a sprinkler during 6 hours (watered area). The plot is entirely laid to grass. 


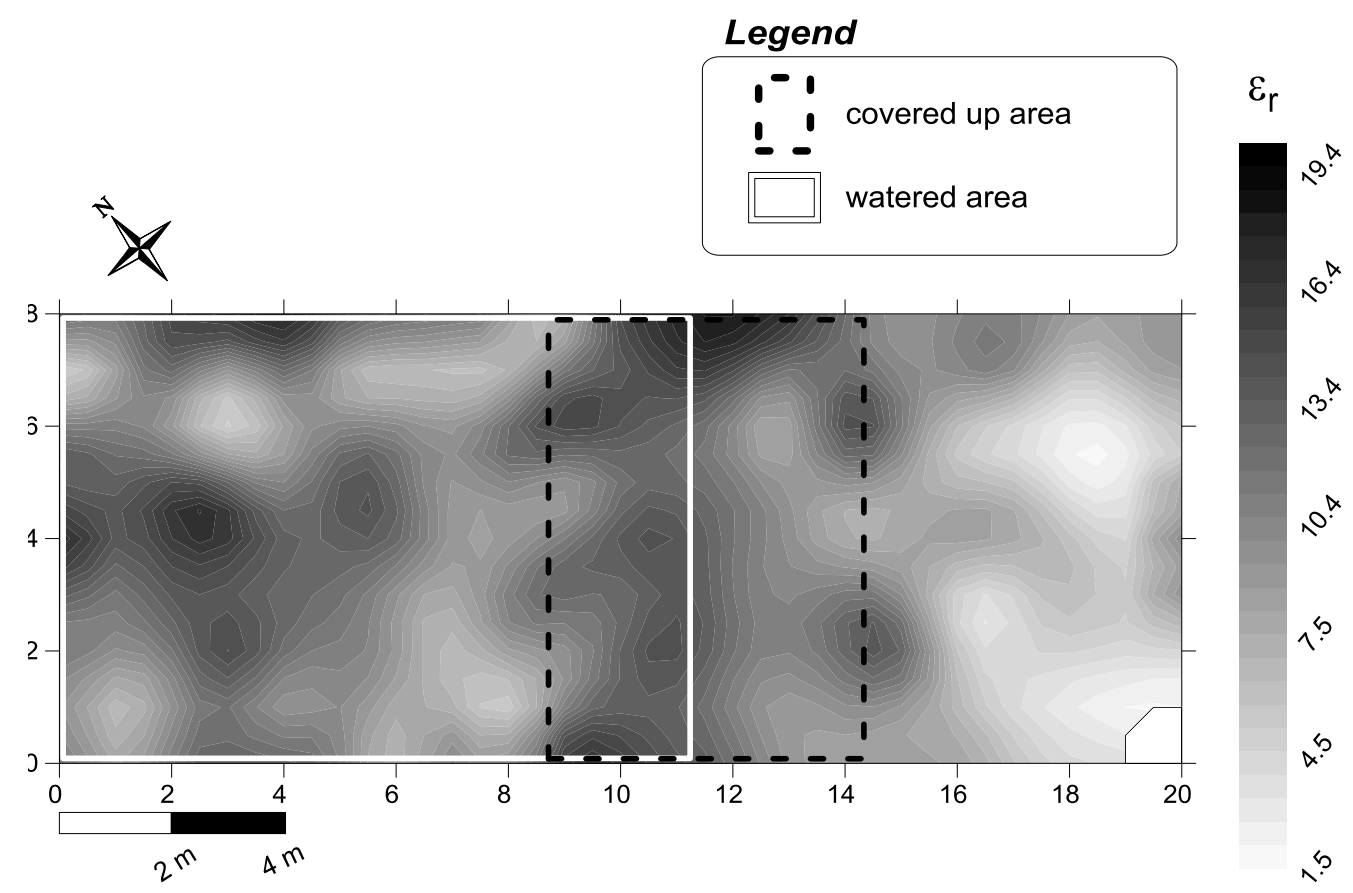

659 Figure 7: Map of the dielectric permittivity of sandy alluvia, measured with the CE120 on a $1 \mathrm{~m}$ grid 660 mesh. Artificial water content contrasts were created using a $6 \times 8 \mathrm{~m}$ canvas sheet during 6 months 661 (covered up area) and a sprinkler during 6 hours (watered area). The plot is entirely laid to grass. 
a)

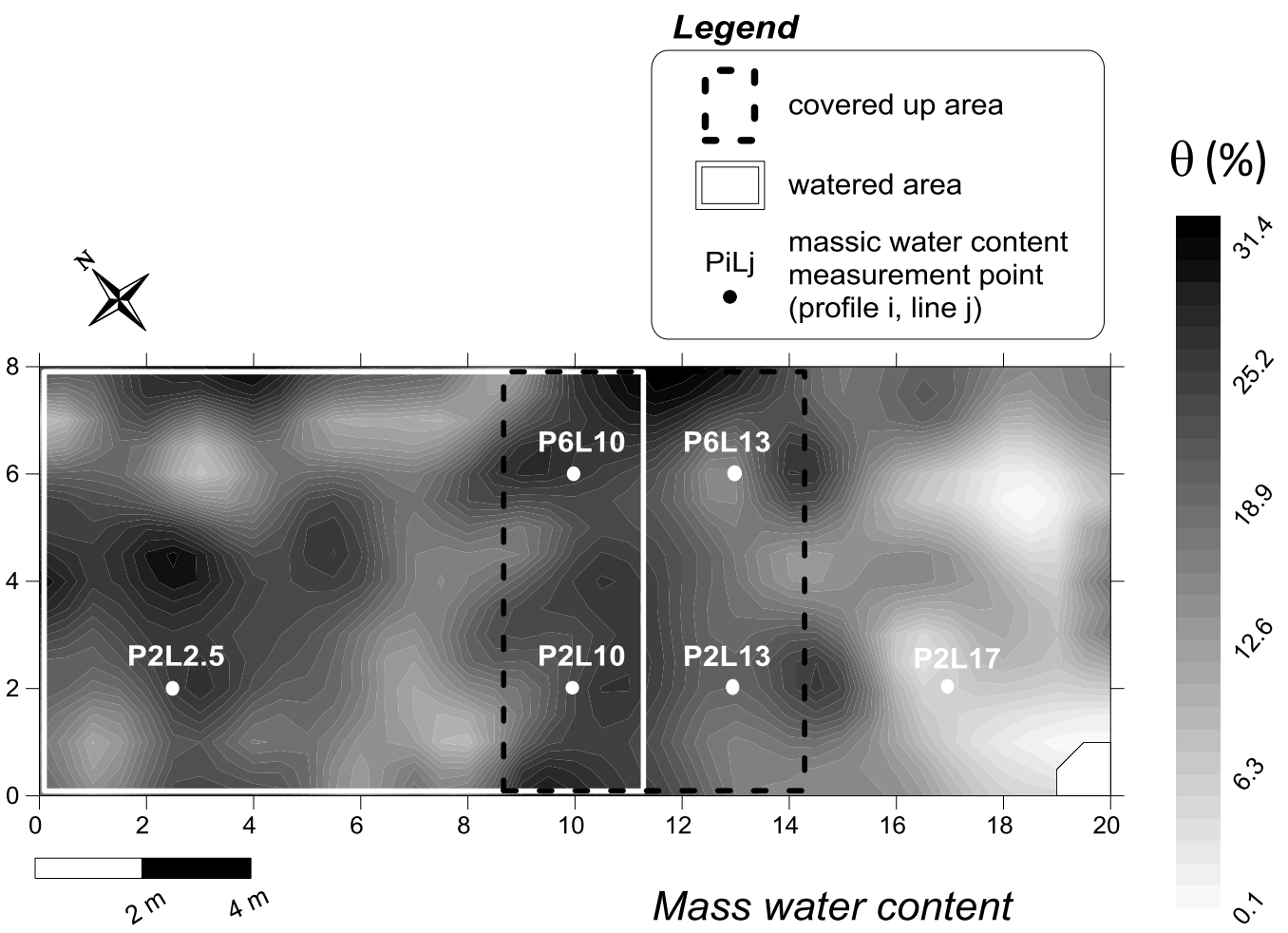

b)

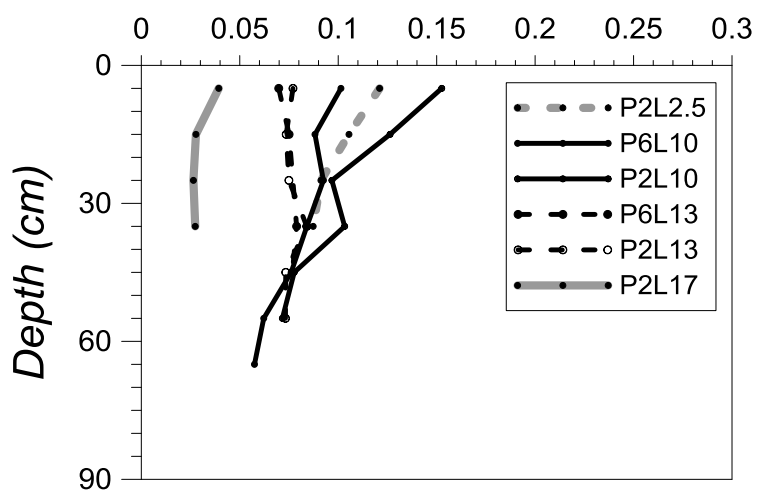

Figure 8: a) Map of the volumetric water content of sandy alluvia, determined using the CE120 measurements and the Topp equation (Topp et al., 1980) on a $1 \mathrm{~m}$ grid mesh. Artificial water content contrasts were created using a $6 \times 8 \mathrm{~m}$ canvas sheet during 6 months (covered up area) and a sprinkler during 6 hours (watered area). The plot is entirely laid to grass. b) Mass water content measured at the Pilj location points on the map a) as a function of the studied sample depth. 
March 2011

a) $A M=0.5 \mathrm{~m}$

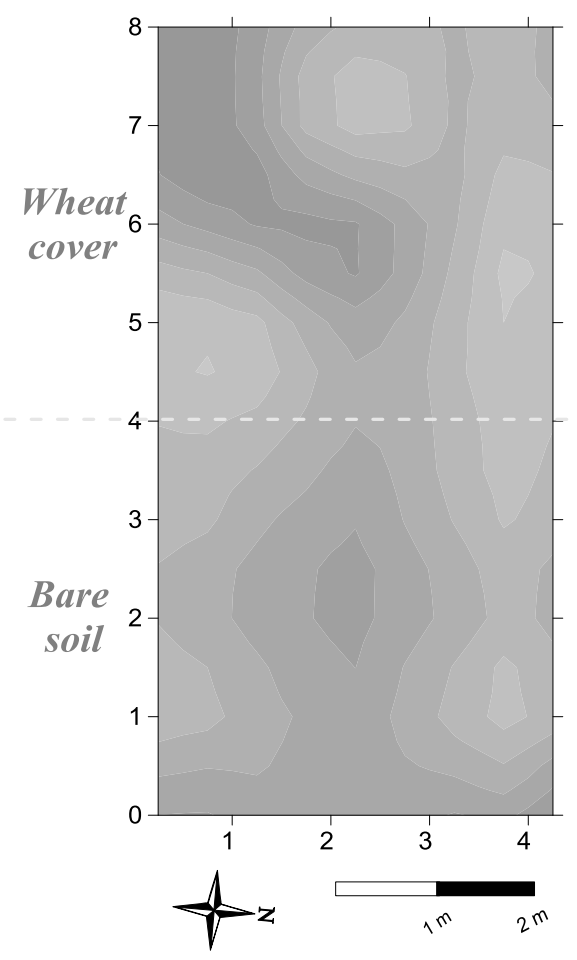

b) $A M=1 \mathrm{~m}$

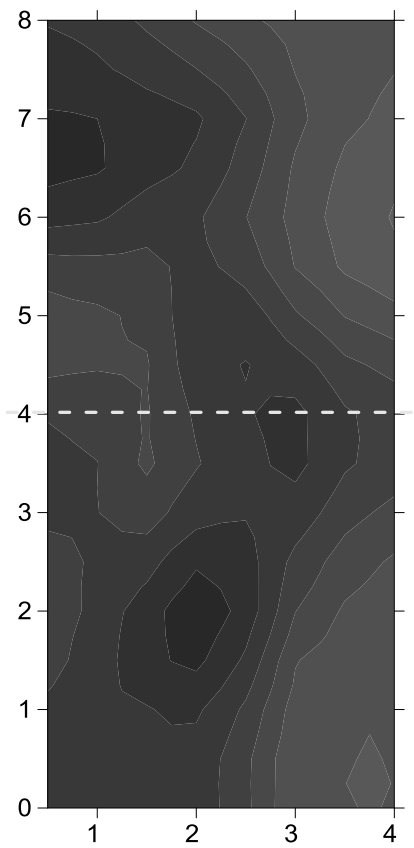

May 2011

d) $a=1 \mathrm{~m}$

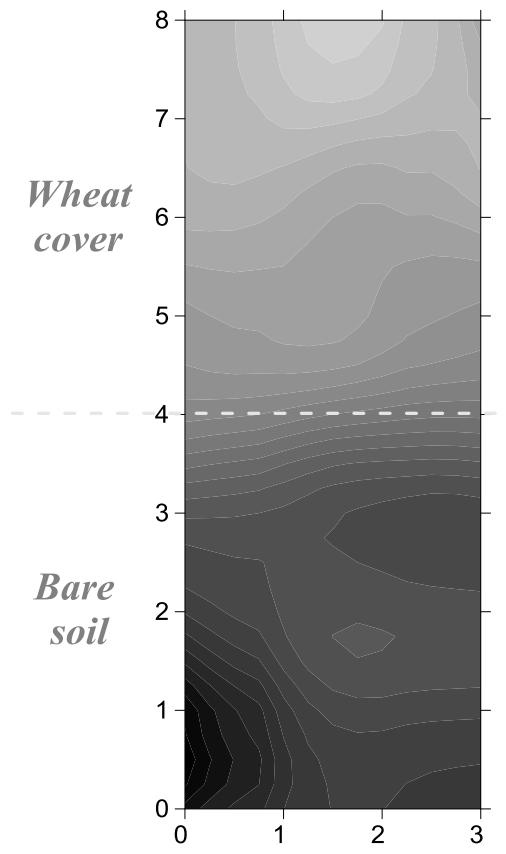

c) $A M=1.5 \mathrm{~m}$

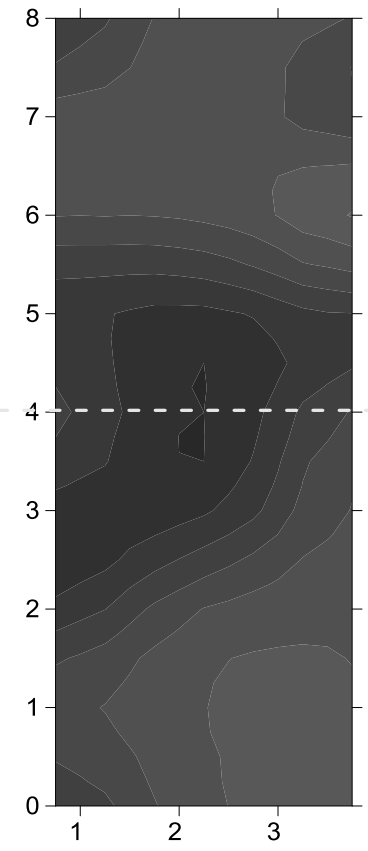

673 Figure 9: Maps of the electrical resistivity of a clay-loam soil, measured with an RM15-D (Geoscan 674 Research) resistivimeter in March 2011 (for a), b) and c)), using a Pole-Pole electrode configuration 675 (a) $A M=0.5 \mathrm{~m}$; b) $A M=1 \mathrm{~m}$; c) $A M=1.5 \mathrm{~m}$ ) on a $0.5 \mathrm{~m}$ grid mesh and an RMCA-4 (CNRS) 676 resistivimeter in May 2011 (for $\mathbf{d})$ ), using a Wenner $\alpha$ electrode configuration $(a=1 \mathrm{~m})$ on a $0.5 \mathrm{~m}$ 677 grid mesh. Wheat has been planted on the western half of the plot, while its eastern part is let bare. 


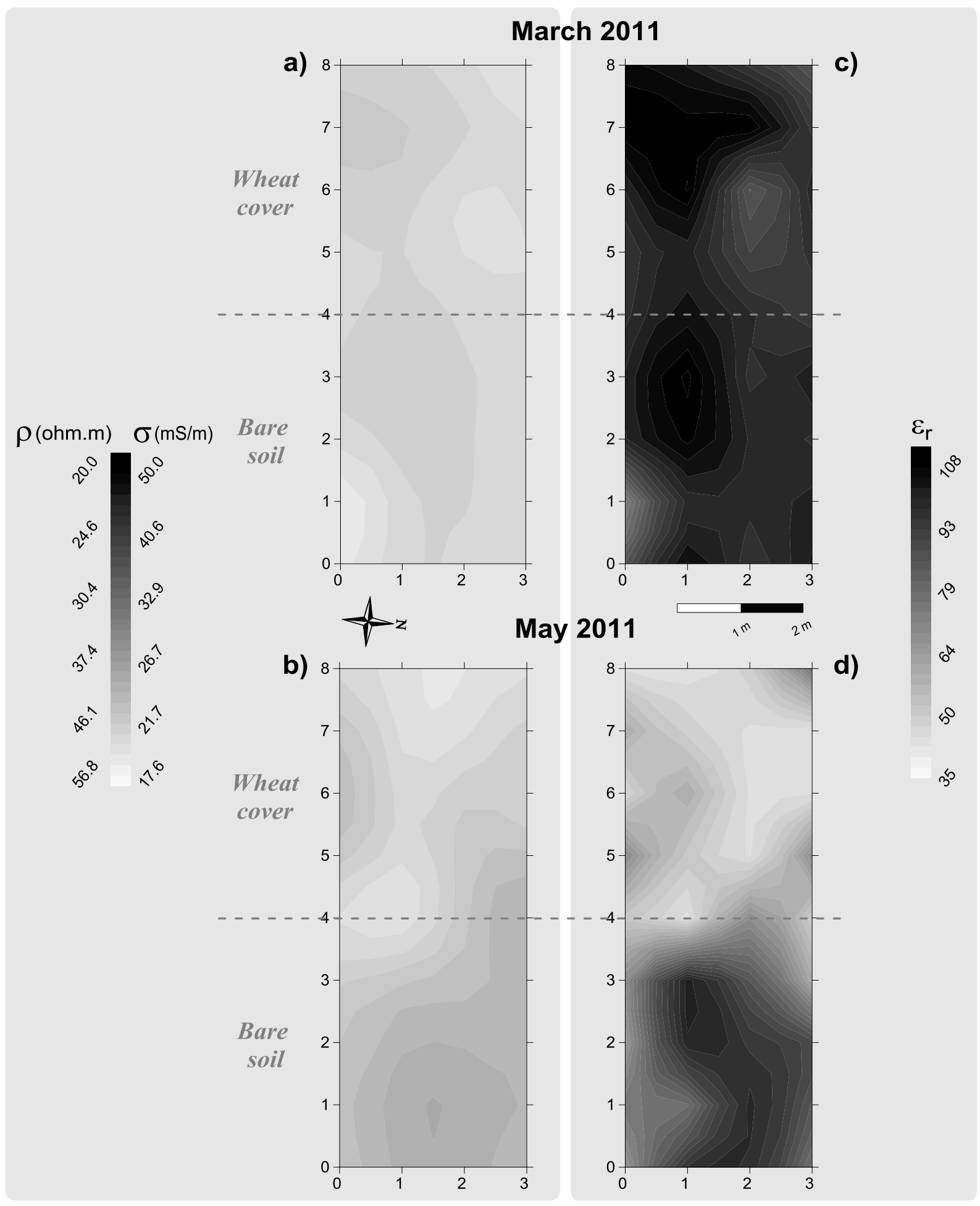

Figure 10: Maps of the electrical conductivity (for a) and b)) and the dielectric permittivity (for c) and 681 d)) of a clay-loam soil, measured with the CE120 in March (for a) and c)) and May (for b) and d)) 2011 on a $0.5 \mathrm{~m}$ grid mesh. Wheat has been planted on the western half of the plot, while its eastern part is let bare. 


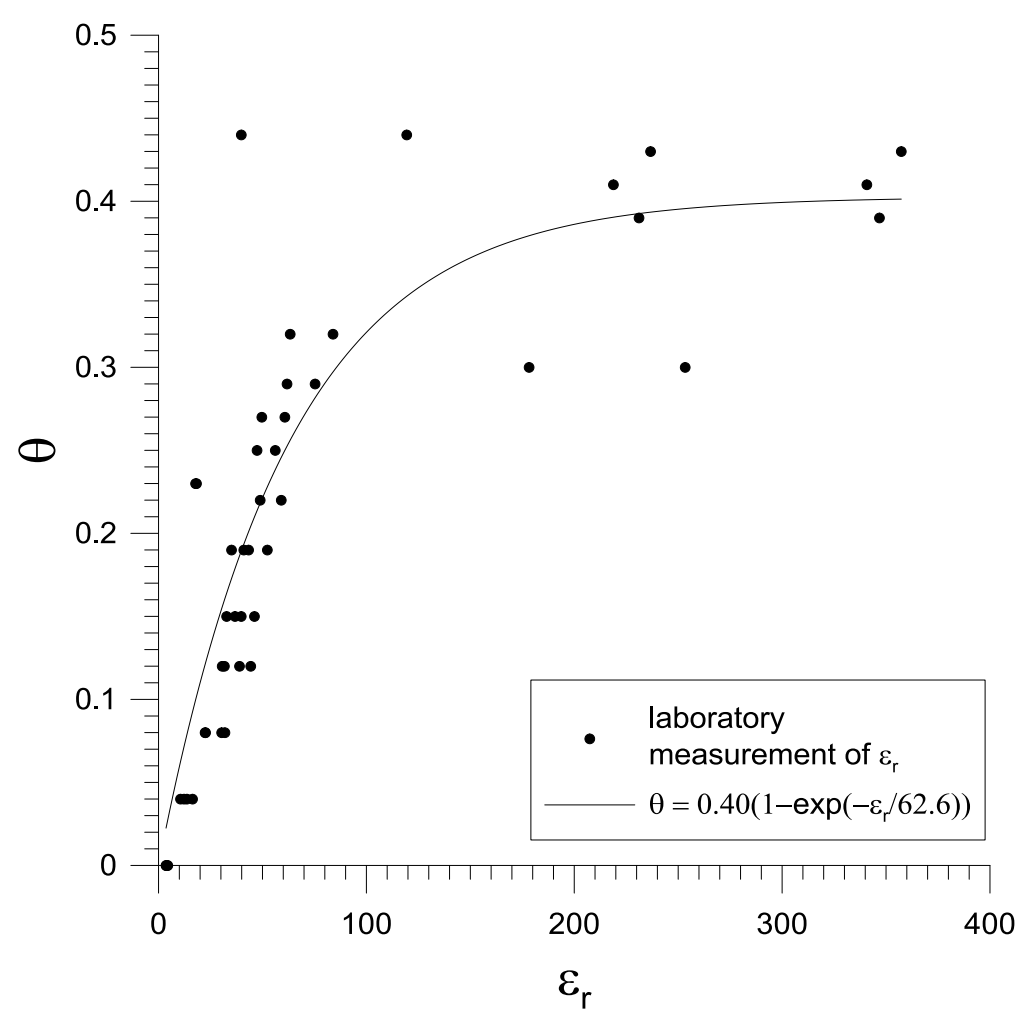

686 Figure 11: Laboratory measurements of the relative dielectric permittivity $\varepsilon_{\mathrm{r}}$ of soil samples from the 687 plot of ORE-ACBB at INRA d'Estrée-Mons for various volumetric water contents $\theta\left(\sigma_{\mathrm{w}}=536 \mu \mathrm{S} / \mathrm{cm}\right.$ $688 \pm 4 \%$ at $25^{\circ} \mathrm{C}$ ). Measurements of the complex dielectric permittivity were made with a capacitive cell 689 coupled to a frequency response analyzer at two different frequencies (1.024MHz and $2.048 \mathrm{MHz})$. A 690 good fit was found for $\theta=0.40\left(1-\exp \left(-\varepsilon_{\mathrm{r}} / 62.6\right)\right)$ with a determination coefficient $R^{2}$ of 0.779 for 47 691 data points. 
a)
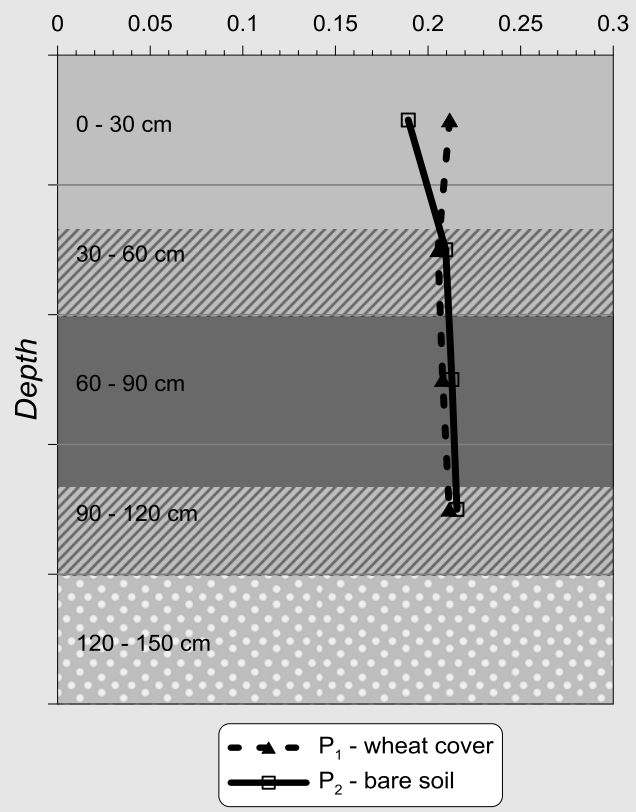

Soil textural characterization

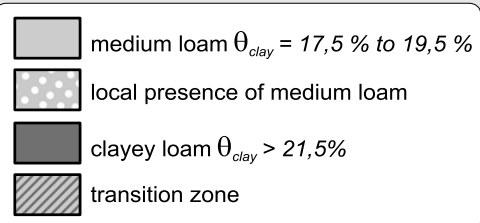

b) Mass water content

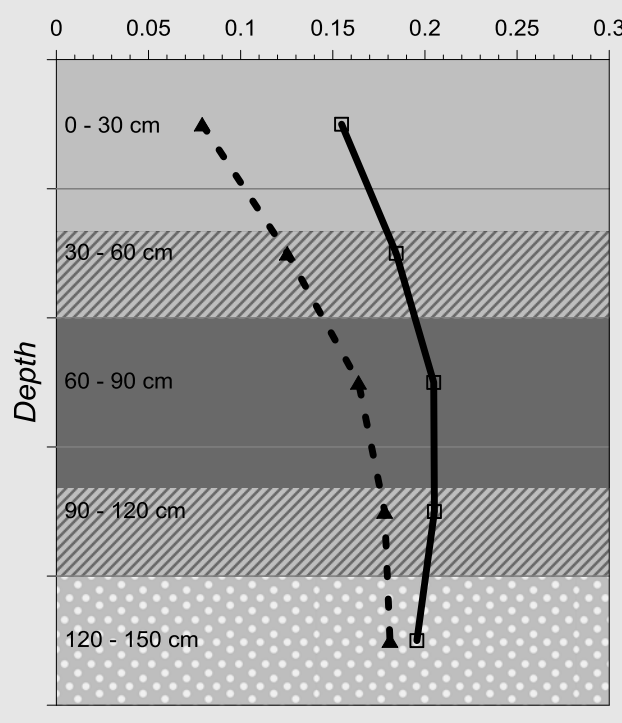

March 2011

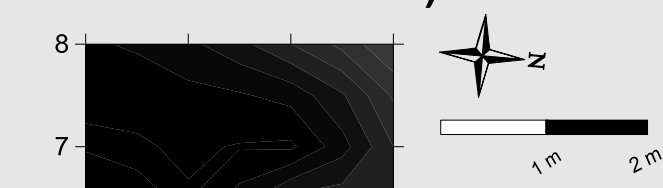

Wheat

cover

Bare

soil

$\theta(\%)$

2

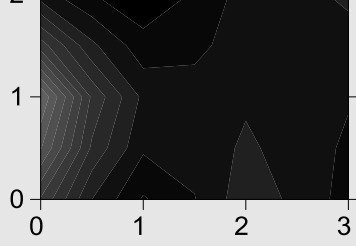

May 2011

d)

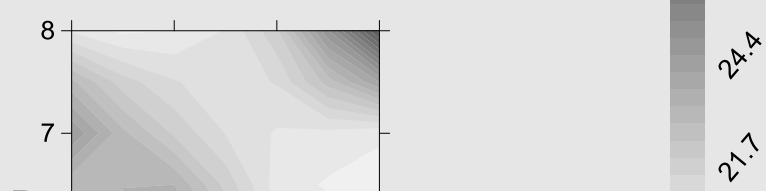

Wheat

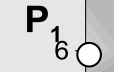

cove

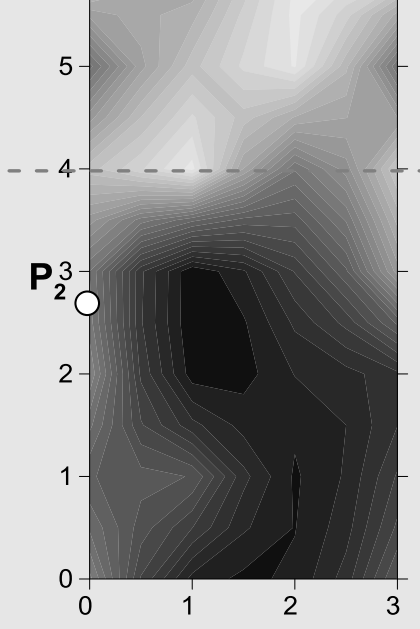

cover

20

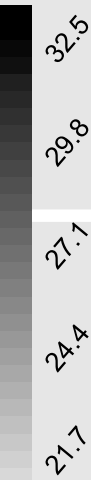

$2^{3^{5}}$

$\hat{\imath}$

$x$

$\uparrow$

Bare

soil

Legend

$\mathrm{P}_{\mathbf{i}} \quad$ massic water content

i measurement point

Figure 12:

695 c) and d): Maps of the volumetric water content of the clay-loam soil, determined using the CE120 696 measurements and laboratory experimentations (Kessouri, 2012) on a 0.5m grid mesh in March (for c)) and May (for d)) 2011. 
698 a) and b): Mass water content measured at the Pi location points on the maps $\mathbf{c}$ ) and d) as a function 699 of the studied sample depth. The soil textural characterization versus depth has been added to the 700 graph.

701 